\title{
Mitochondrial fusion but not fission regulates larval growth and synaptic development through steroid hormone production
}

\author{
Hector Sandoval', Chi-Kuang Yao ${ }^{1 \dagger}$, Kuchuan Chen ${ }^{2}$, Manish Jaiswall,

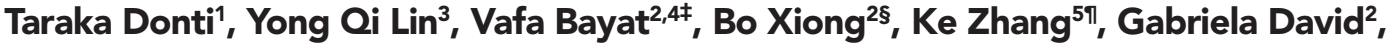 \\ Wu-Lin Charng 1,2, Shinya Yamamoto ${ }^{1,2,6}$, Lita Duraine ${ }^{3}$, Brett H Graham ${ }^{1,2}$, \\ Hugo J Bellen ${ }^{1,2,3,6,7 *}$
}

'Department of Molecular and Human Genetics, Baylor College of Medicine, Houston, United States; ${ }^{2}$ Program in Developmental Biology, Baylor College of Medicine, Houston, United States; ${ }^{3}$ Howard Hughes Medical Institute, Baylor College of

*For correspondence: hbellen@ bcm.edu

Present address: ${ }^{\dagger}$ Institute of Biological Chemistry, Academia Sinica, Taipei, Taiwan:

‡Department of Pathology, Stanford Hospital and Clinics, Stanford, United States; $\S$ Department of Genome Sciences, University of Washington, Seattle, United States; "Department of Neurology, Johns Hopkins University School of Medicine, Baltimore, United States

Competing interests: The authors declare that no competing interests exist.

Funding: See page 18

Received: 02 June 2014

Accepted: 13 October 2014

Published: 14 October 2014

Reviewing editor: Richard J Youle, National Institute of Neurological Disorders and Stroke, National Institutes of Health, United States

(c) Copyright Sandoval et al. This article is distributed under the terms of the Creative Commons Attribution License, which permits unrestricted use and redistribution provided that the original author and source are credited.
Medicine, Houston, United States; ${ }^{4}$ Medical Scientist Training Program, Baylor College of Medicine, Houston, United States; ${ }^{5}$ Program in Structural and Computational Biology and Molecular Biophysics, Baylor College of Medicine, Houston, United States; ' ${ }^{6}$ Jan and Dan Duncan Neurological Research Institute, Texas Children's Hospital, Houston, United States; ${ }^{7}$ Department of Neuroscience, Baylor College of Medicine, Houston, United States

Abstract Mitochondrial fusion and fission affect the distribution and quality control of mitochondria. We show that Marf (Mitochondrial associated regulatory factor), is required for mitochondrial fusion and transport in long axons. Moreover, loss of Marf leads to a severe depletion of mitochondria in neuromuscular junctions (NMJs). Marf mutants also fail to maintain proper synaptic transmission at NMJs upon repetitive stimulation, similar to Drp1 fission mutants. However, unlike Drp1, loss of Marf leads to NMJ morphology defects and extended larval lifespan. Marf is required to form contacts between the endoplasmic reticulum and/or lipid droplets (LDs) and for proper storage of cholesterol and ecdysone synthesis in ring glands. Interestingly, human Mitofusin-2 rescues the loss of LD but both Mitofusin-1 and Mitofusin-2 are required for steroidhormone synthesis. Our data show that Marf and Mitofusins share an evolutionarily conserved role in mitochondrial transport, cholesterol ester storage and steroid-hormone synthesis.

DOI: 10.7554/eLife.03558.001

\section{Introduction}

Mitochondrial dynamics plays a critical role in the control of organelle shape, size, number, function and quality control of mitochondria from yeast to mammals (Westermann, 2009; Chan, 2012). It consists of fusion and fission of mitochondria, which are regulated by several GTPases (van der Bliek et al., 2013). Mitochondrial fusion requires the fusion of the outer membrane followed by inner membrane fusion (Chan, 2012; Mishra et alo, 2014). In mammals, Mitofusin 1 (Mfn1) and Mitofusin 2 (Mfn2) regulate outer mitochondrial fusion whereas inner membrane fusion is controlled by Optic atrophy protein 1 (Opa1). Mitochondrial fission is regulated by Dynamin related protein 1 (Drp1) (van der Bliek et al., 2013). Decreased fusion results in fragmented round mitochondria, while defective fission leads to fused and enlarged mitochondria (van der Bliek et al., 2013). 
eLife digest Mitochondria are the main source of energy for cells. These vital and highly dynamic organelles continually change shape by fusing with each other and splitting apart to create new mitochondria, repairing and replacing those damaged by cell stress.

For nerve impulses to be transmitted across the gaps (called synapses) between nerve cells, mitochondria need to supply the very ends of the nerve fibers with energy. To do this, the mitochondria must be transported from the main body of the nerve cell to the tips of the nerve fibers. This may not happen if mitochondria are the wrong shape, size or damaged.

While searching for genetic mutations that disrupt nerve function in the fruit fly Drosophila, Sandoval et al. spotted mutations in a gene called Marf. Further investigations revealed that flies with mutant versions of Marf have small, round mitochondria, and their nerves cannot transmit signals to muscles when they are highly stimulated. This is because the mutant mitochondria are not easily transported along nerve fibers, and so not enough energy is supplied to the synapses. The synapses of the Marf mutants are also abnormally shaped. Sandoval et al. found that this is not because Marf is lost in the neurons themselves, but because it is lost from a hormone-producing tissue called the ring gland.

Another problem found in flies with mutated Marf genes is that they stop developing while in their larval stage. Sandoval et al. established that this could also be related to the loss of Marf from the ring gland. The Marf protein has two different functions in the ring gland: forming and storing droplets of fatty molecules used in hormone production, and synthesising a hormone that controls when a fly larva matures into the adult fly. This suggests that the lower levels of this hormone produced by Marf mutant flies underlies their prolonged larval stages and synapse defects.

Vertebrates (animals with backbones, such as humans) have two genes that are related to the fly's Marf gene. When the human forms of these genes were introduced into mutant flies that lack a working copy of Marf, hormone production was only restored if both genes were introduced together. This indicates that these genes have separate roles in vertebrates, but that these roles are both performed by the single fly gene.

The role of Marf in tethering mitochondria in the ring gland may allow us to better understand how this process affects hormone production and how the different parts of the cell communicate. DOI: 10.7554/eLife.03558.002

Loss of these mitochondrial GTPases results in lethality in worms, flies and mice (Chen et al., 2003; Westermann, 2009; Debattisti and Scorrano, 2012). Mutations in the human DRP1 gene causes a dominant fatal infantile encephalopathy associated with defective mitochondrial and peroxisomal fission (Waterham et al., 2007). On the other hand, missense mutations in OPA1 lead to a dominant optic atrophy (Alexander et al., 2000; Delettre et al., 2000). Depending on the severity of the mutation, patients may also suffer from ataxia and neuropathy (Yu-Wai-Man et al., 2010). Also, missense mutations in MFN2 cause Charcot-Marie-Tooth type 2A, a common autosomal dominant peripheral neuropathy associated with axon degeneration (Zuchner et al., 2004). Finally, aberrant levels of mitochondrial GTPases have been associated with Parkinson's, Huntington's and Alzheimers' diseases (Itoh et al., 2012). These observations in model organisms and human patients suggest that mitochondrial dynamics affects neuronal maintenance in many different contexts.

A significant imbalance of mitochondrial fission and fusion may affect the subcellular distribution of mitochondria, especially in neurons since they need to efficiently traffic from the soma to the synapses (Sheng, 2014). Loss of Drosophila Drp1 impairs the delivery of mitochondria to neuromuscular junctions (NMJs), likely because they are large and interconnected. This defect is also associated with a severe depletion of mitochondria in NMJs, which affects local ATP production. This in turn affects the trafficking of synaptic vesicles upon endocytosis during prolonged stimulation (Verstreken et al., 2005). Similarly, in vertebrates, loss of Drp1 leads to an accumulation of mitochondria in the soma and reduced mitochondrial density in dendrites of hippocampal neurons (Li et al., 2004). The Drp1 data in flies and vertebrates indicate that the expanded size of mitochondria affects their mobility (Sheng, 2014).

Mitochondrial trafficking may also be affected by the physical interaction between the mitochondria and the transport machinery. Recent studies have documented a direct interaction between Mfn2 and a motor adaptor complex for mitochondrial transport, Miro2 (Misko et al., 2010). Moreover, loss of MFN2 in Purkinje cells displayed reduced mitochondrial motility in cerebellar dendrites (Chen et al., 
2007) and reduced mitochondrial transport in axons in cultured dorsal root ganglion neurons (Misko et al., 2010). These data suggest that an interaction of Mfn2 with Miro2 may be important for its role in trafficking (Misko et alo, 2010). Although loss of both Drp1 and MFN2 impair mitochondrial trafficking, a careful comparison of the phenotypes associated with loss of Drosophila Drp1, Mitofusin or Marf, would be useful as the suggested mechanisms by which they impair transport seem very different.

In addition to their roles in fission and fusion, Drp1, Mfns and Opa1 have been implicated in a variety of other processes. For example, Drp1 has been shown to facilitate the induction of apoptosis (Frank et al., 2001) whereas Opa1 was shown to affect the stability of cristae junction in inner mitochondrial membrane (Frezza et al., 2006). Finally, Mfn2 also tethers mitochondria to the endoplasmic reticulum (ER) to mediate $\mathrm{Ca}^{2+}$ uptake (de Brito and Scorrano, 2008). However, the molecular mechanisms underlying these non-canonical functions are less well studied.

In an unbiased screen designed to identify essential genes that affect neuronal function (Yamamoto et al., 2014), we identified the first mutant allelic series of Marf in Drosophila. Here we exploit these mutants to determine how loss of Marf affects mitochondrial transport when compared to Drp1 loss. Surprisingly, we observe NMJ defects only in Marf mutants but not in Drp1 mutants. These defects are regulated non-cell autonomously by steroid-hormones produced in ring glands (RG), a major endocrine organ in insects. Through expression of human MFN1 or MFN2 in Marf mutant RG, we show that MFN1 and MFN2 have both distinct and complementary roles.

\section{Results}

\section{Marf affects mitochondrial distribution in photoreceptors}

Through a forward genetic screen on the Drosophila X-chromosome (Yamamoto et al., 2014) we isolated seven independent lethal alleles of Marf that affect electroretinogram (ERG) recordings in homozygous mutant clones (Figure 1A,C, Figure 1-figure supplement 1). The on- and off-transients (Figure 1A, red arrows) of the ERG are a read-out of synaptic transmission between photoreceptors (PR) and postsynaptic cells, while the amplitude of the depolarization (Figure 1A, green bracket) is a measure of the function of the phototransduction cascade (Wang and Montell, 2007). The Marf mutations vary in strength (Figure 1A,E and Figure 1-figure supplement 1B), providing an allelic series. ERG recordings in homozygous mutant eye clones reveal a reduction in on- and off-transients as well as loss of amplitude in one day old flies (Figure 1A). The ERG recordings differ from Drp1 mutants that only exhibit a loss of on- and off-transients but a normal amplitude (Figure 1B, [Verstreken et al., 2005]). In summary, loss of Marf severely impairs the phototransduction cascade as well as synaptic transmission, whereas loss of Drp1 mainly affects synaptic transmission of PRs.

Lethal staging shows that most Marf mutants $(A, B, E, F$ and $G$ ) die as third instars after a very extended larval stage period of 18-21 days, which typically takes 6 days in wild type animals (Figure 1-figure supplement 1B). The lethality of all Marf mutants is rescued by a Marf genomic DNA construct or by a ubiquitously expressed Marf cDNA (Figure 1-figure supplement 1A), showing that the Marf mutations are responsible for the lethality (Figure 1-figure supplement 1B). Moreover, transheterozygous Marf ${ }^{B} / D f(1)$ Exel6239 female mutants display the same lethal phase as Marf $f^{B} / Y$ males, suggesting that Marf $^{\beta}$ is likely to be a severe loss of function allele or null allele (Figure 1-figure supplement 1B). Finally, Marf $f^{B}$ hemizygous males exhibit a severe protein loss compared to Marf ${ }^{G}$ hemizygous males and controls (Figure 1-figure supplement 1C), suggesting that this missense mutation in the GTPase domain (Figure 1C) also destabilizes the protein.

Since mitochondrial transport has been shown to be affected in some neurites of MFN2-deficient vertebrate cells (Chen et al., 2007), we performed Transmission Electron Microscopy (TEM) at the PR terminals. Marf mutants exhibit a very severe loss of mitochondria (Figure 1D, yellow arrows) in PR terminals when compared to control (Figure 1D,E). The severity of the loss of mitochondria (Figure 1E) correlates with the loss of neuronal function gauged by ERGs (Figure 1A). These data are reminiscent of the documented lack of mitochondria in PR terminals in Drp1 mutants (Verstreken et alo, 2005). However, the mitochondria in Marf mutant PRs are significantly smaller in size than controls (Figure 1D, yellow arrows), suggesting that an active transport mechanism is impaired.

\section{Marf affects mitochondrial function and distribution in NMJs}

To assess if mitochondrial size is also affected in mutant muscles, we stained Marf and Drp1 (Figure 2source data 1) mutants with an anti-mitochondrial complex V antibody (ATP5A) (Baqri et al., 2009). 


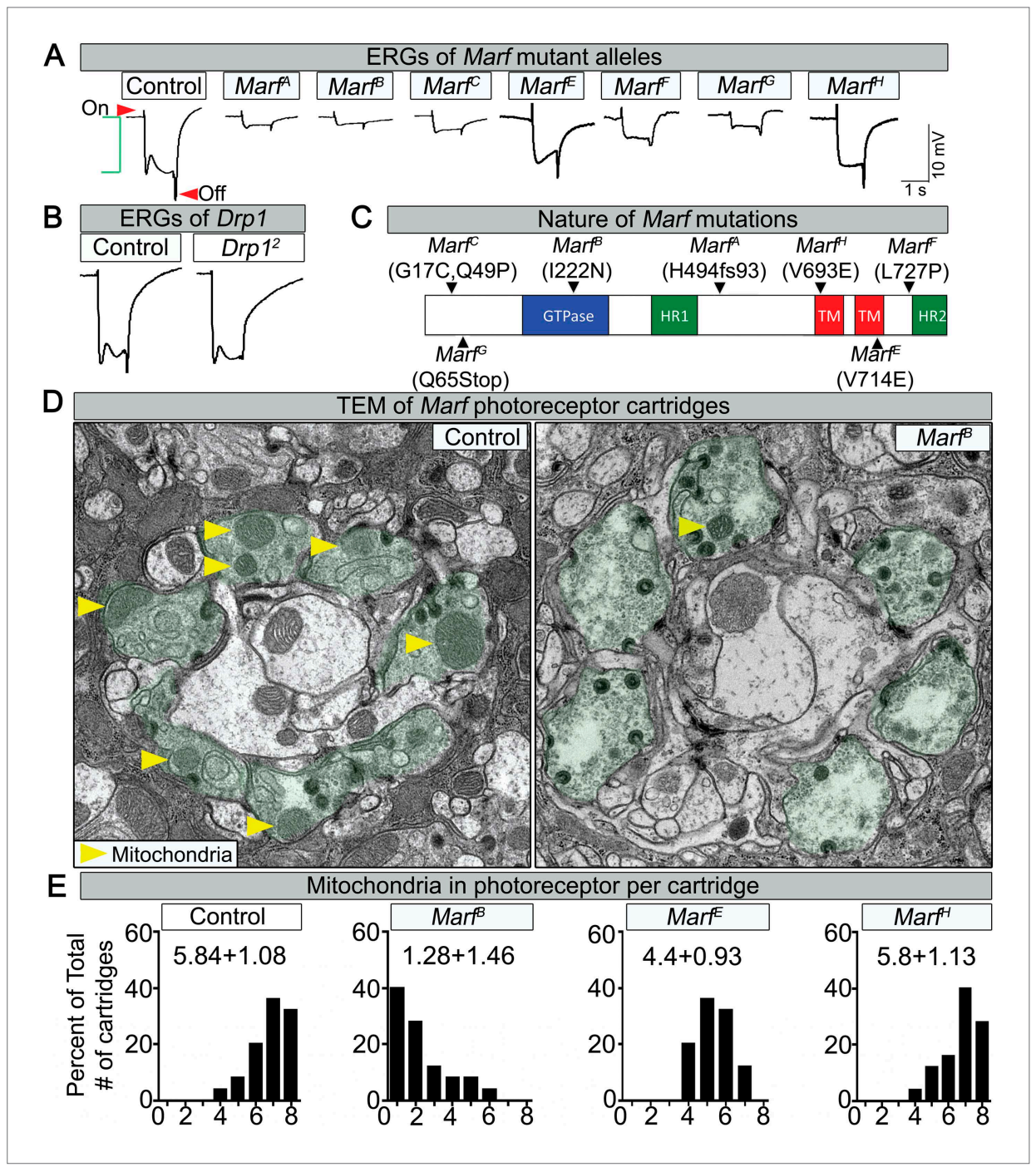

Figure 1. Loss of Marf impairs phototransduction and affects mitochondrial localization to photoreceptor terminals. (A) Electroretinograms (ERGs) of 1 day old ey-FLP mutant clones of 7 different Marf mutants or isogenized wild type clones (Control). ERGs of Marf mutant alleles and control flies. A typical ERG trace is comprised of an on-transient (red arrow), a depolarization (green bracket) and an off-transient (red arrow). (B) ERGs of Drp1 mutants and control flies. (C) Marf protein domains and localization of EMS-induced mutations of the seven Marf mutant alleles identified by sequencing. $\mathrm{H} 494 \mathrm{fs} 93$ = insertion of an $\mathbf{A}$ at nucleotide codon for amino acid H494 that generates 93 new amino acids followed by a premature stop codon. TM = transmembrane domain. HR = heptad repeat. (D) TEM sections of a cartridge containing fly photoreceptor terminals (green shading). Marf mutant photoreceptor terminals display reduced number and size of mitochondria (yellow arrow heads) compared to Marf-genomic rescue controls. (E) Quantification of total mitochondria number per cartridge in Marf mutants and Marf-genomic rescue photoreceptor terminals (Control). 50 cartridges per genotype.

DOI: 10.7554/eLife.03558.003

The following figure supplement is available for figure 1:

Figure supplement 1. Mapping, lethal staging and Marf protein expression of Marf mutant alleles. DOI: 10.7554/eLife.03558.004

As expected, Drp1 mutants have filamentous mitochondria whereas Marf mutants have small, rounded mitochondria (Figure 2A and Figure 2-source data 2). However, both Marf and Drp1 mutant mitochondria produce similar reduced levels of ATP when compared to controls (Figure $2 \mathrm{C}$ and Figure 2-source data 2). Interestingly, the mitochondrial membrane potential (MMP) of Drp1 mutants as measured with tetramethylrhodamine ethyl ester (TMRE) (Scaduto and Grotyohann, 1999) is slightly elevated, as reported before (Verstreken et al., 2005), when compared to controls whereas MMP of Marf 

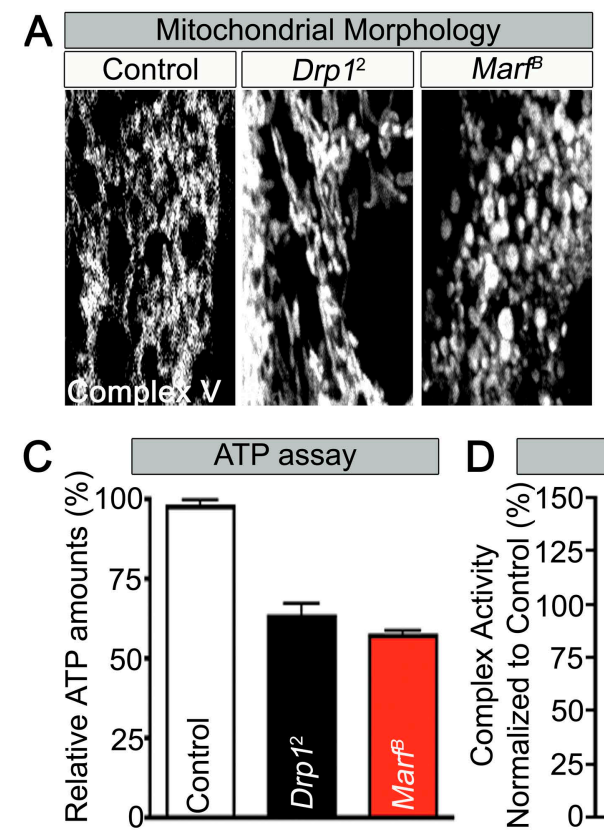

B

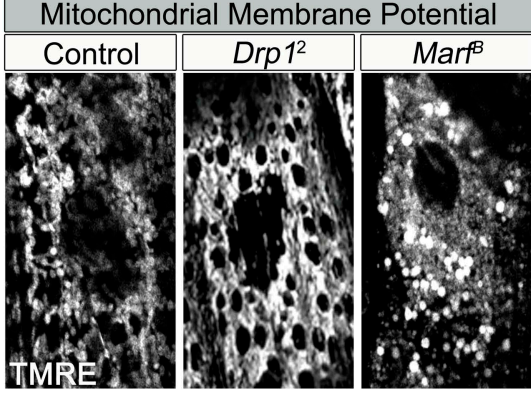

C

E

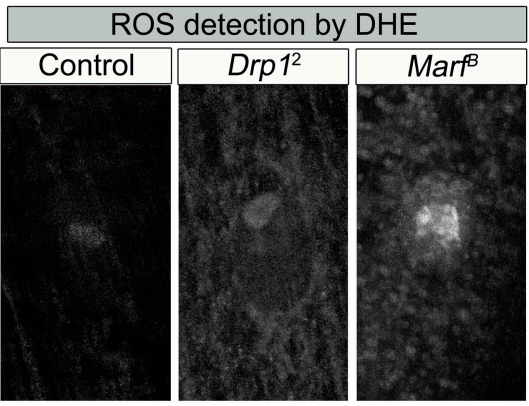

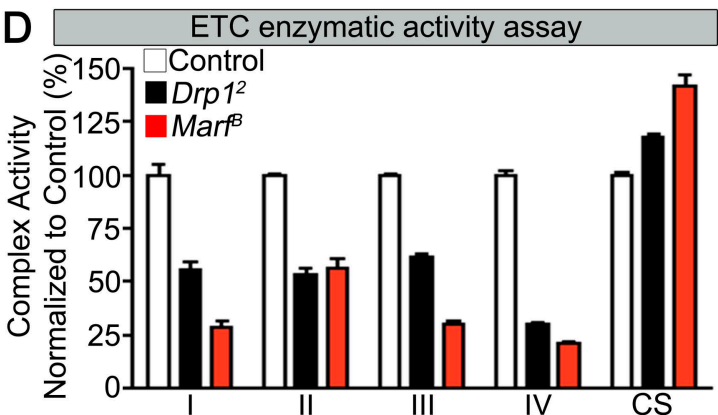

$F$

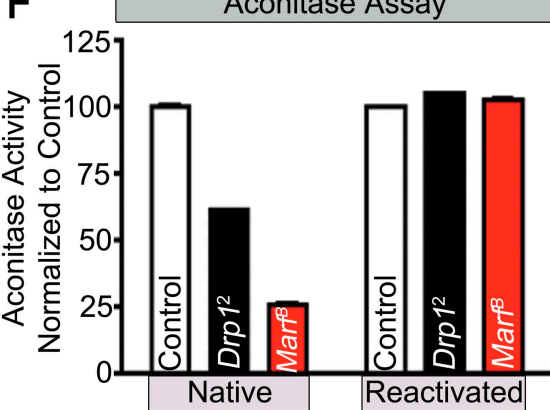

Figure 2. Mitochondrial morphology and function in Marf and Drp1 mutants. (A) Mitochondrial morphology based on anti-Complex $\mathrm{V}$ antibody staining (Complex V) in larval muscles (Zoom in view around muscle nucleus). (B) Mitochondrial membrane potential as measured by the TMRE dye in larva muscle. (C) Relative ATP amounts. (D) Measurement of the enzymatic activity of electron transport chain (ETC) complexes (I-IV) from purified mitochondria from third instar larvae. All the ETC activities were normalized to citrate synthase (CS) activity of controls. (E and F) ROS is measured by two methods: (E) by DHE staining in larval muscles and (F) by measuring aconitase activity reduction from purified mitochondria. Reducing reagents reactivate native aconitase. Aconitase activities were normalized to controls. (C, $\mathbf{D}$ and $\mathbf{F}$ ) error bars represent $\pm \mathrm{SEM}$.

DOI: 10.7554/eLife.03558.005

The following source data are available for figure 2:

Source data 1. Lethal staging of Drp1 mutants.

DOI: 10.7554/eLife.03558.006

Source data 2. Phenotypic comparison of Marf, Drp1 and Marf and Drp1 mutants. DOI: 10.7554/eLife.03558.007

mutants is reduced (Figure 2B and Figure 2-source data 2). Measurements of the activity of the Electron Chain Complexes (ETC I, II, III and IV) that pump protons across the mitochondrial inner membrane from the mitochondrial matrix to the inner membrane space to generate the MMP revealed that all ETC complex activities are similarly or more severely affected in Marf than Drp1 mutants (Figure 2D). Furthermore, measurement of reactive oxygen species (ROS) by dihydroethidium (DHE) staining (Shidara and Hollenbeck, 2010) and mitochondrial aconitase assay (native activity of aconitase negatively correlates with ROS levels) (Yan et al., 1997) shows that Marf mutants are significantly more 
severely affected than Drp1 mutants (Figure 2E,F and Figure 2-source data 2). The ROS data is in agreement with the ETC data as loss of function of $\mathrm{Cl}$ and $\mathrm{CIII}$ are considered the major drivers of increased ROS (Koopman et al., 2013). In summary, Marf and Drp1 mutants exhibit dysfunctional mitochondria, but loss of Marf affects their function more severely.

Loss of one copy of MFN2 in human causes a progressive and severe loss of function of neurons with long axons and affects motor neurons (MN) more severely than sensory neurons (Zuchner et al., 2004). To assess if mitochondria in $M N$ are affected in larvae we expressed MitoGFP in $M N$ using the D42-Gal4 driver (Pilling et alı, 2006). In the ventral nerve cord (VNC) of control larvae, MitoGFP mostly localizes to the neuropil (Figure 3A). Marf mutants show an obvious reduction in levels of mitochondria in the neuropil and the mitochondria mostly form clumps in the soma and the initial segments of axons (Figure 3A). In control MN, MitoGFP also labels numerous mitochondria in axons that innervate proximal (A3) and more distal (A5) segments (Figure 3B). In the axons of Marf mutants, fewer MitoGFP-marked mitochondria are observed in distal axons compared to controls (Figure 3B). These data show that loss of Marf impairs, but does not abolish, axonal mitochondrial transport (Figure 3B).

To assess the presence of mitochondria at NMJs, we counted MitoGFP positive puncta in boutons labeled by anti-Discs Large 1 (Dlg1 [Parnas et al., 2001]). While control NMJs contain numerous mitochondria per bouton, Marf boutons contain almost no mitochondria, even fewer than in Drp1 mutants (Figure 3C, see Figure legend, [Verstreken et al., 2005]). However, unlike Drp1 mutants, Marf mutant NMJs exhibit severe morphological defects (see below). Interestingly, we find no obvious labeling defects with the presynaptic active zone marker Bruchpilot (Wagh et al., 2006), endocytic markers such as $\alpha$-Adaptin (Gonzalez-Gaitan and Jackle, 1997), Dap160 (Roos and Kelly, 1998), Endophilin (Verstreken et al., 2002), and Synaptojanin (Verstreken et al., 2003), or the postsynaptic Glutamate receptor IIA (Qin et al., 2005) in Marf mutants (Figure 3-figure supplement 1). Expression of Marf protein in MN using the D42-Gal4 driver rescues the trafficking defect and restores the presence of mitochondria at the NMJ (Figure 3). However, it does not restore the morphological defects (Figure 3C), suggesting that Marf's function in mitochondrial trafficking is cell autonomous and that the defects in synapse morphology are cell non-autonomous.

Recently, mammalian MFN2 was shown to physically interact with MIRO2, an adaptor protein for motor proteins required for mitochondrial trafficking (Misko et al., 2010). Drosophila miro (dmiro) mutants are severely impaired in mitochondrial trafficking in the VNC (Guo et al., 2005). Indeed, RNAi knockdown of dmiro almost abolishes the presence of mitochondria in axons, a phenotype that is much more severe than what we observe in Marf mutants (data not shown). Moreover, loss of dmiro in Marf mutant MNs largely enhances the mitochondrial trafficking defect in the VNC and proximal axons (Figure 3-figure supplement 2A,B). This suggests that Marf cannot be the sole anchor that binds dMiro for mitochondrial trafficking.

\section{Marf is required to maintain synaptic transmission upon repetitive stimulation}

Loss of mitochondria at NMJs in Drp1 mutants was shown to affect synaptic transmission at high frequency stimulation (Verstreken et al., 2005). To gauge how loss of Marf affects synaptic transmission we performed electrophysiological recordings at the NMJs, using a transheterozygous Marf ${ }^{B} / M^{2} f^{E}$ allelic combination in order to compare larvae of the same size since Marf ${ }^{B}$ mutant are small in size. When stimulated at $0.2 \mathrm{~Hz}$, Marf mutants do not exhibit any obvious defect in transmitter release based on excitatory junction potential (EJP) recordings (Figure 4A). Moreover, the amplitude of spontaneous release events or miniature EJPs (mEJPs) and quantal content are not altered in Marf mutants (Figure 4A). Hence, the average number of vesicles released in response to low frequency stimulations in Marf mutants is not different from Marf genomic-rescue controls. However, Marf mutant terminals are unable to properly sustain a $10 \mathrm{~Hz}$ stimulus for $10 \mathrm{~min}$ when compared to controls (Figure 4B) as the EJP amplitudes progressively decrease. A rundown of synaptic transmission is often observed in endocytic mutants such as endophilin and synaptojanin (Verstreken et al., 2002, 2003; Dickman et al., 2005), dap160 and eps15 (Koh et al., 2004, 2007), and flower (Yao et alı, 2009). We therefore assessed if endocytosis is impaired and used FM1-43, a dye that reversibly binds membranes and is internalized into vesicles (Verstreken et al., 2008). Unlike eps 15 mutants that serve as a positive control, nerve stimulation at $60 \mathrm{mM} \mathrm{K}^{+}$in the presence of FM1-43 effectively labels synaptic boutons in Marf mutants similar to controls (Figure 4C,D). Hence, vesicle endocytosis or evoked responses at $0.2 \mathrm{~Hz}$ are not affected in Marf mutants. These features are similar to Drp1 mutants, suggesting that lack of 


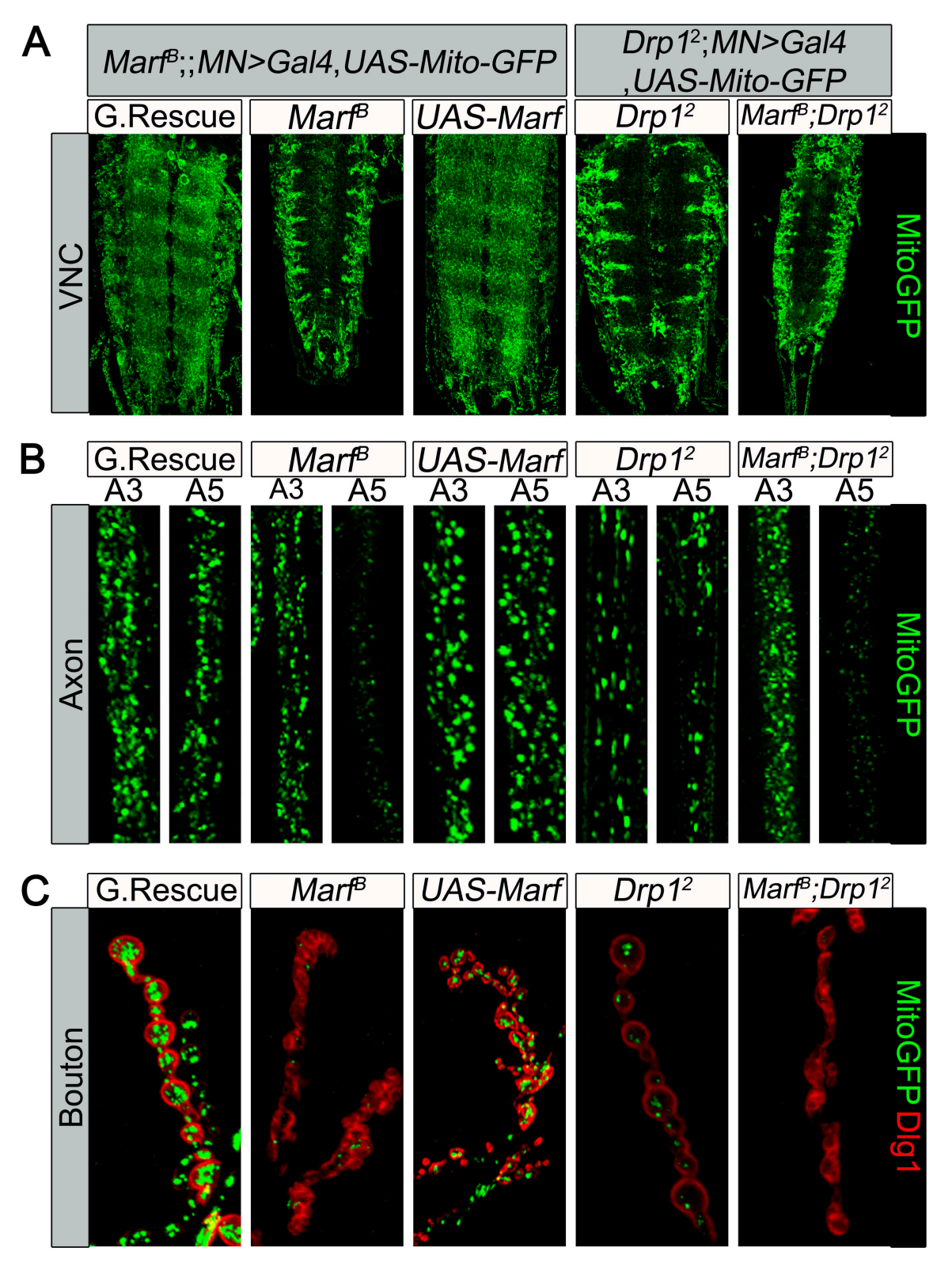

Figure 3. Mitochondrial trafficking defects in distal axons and boutons. Mutations and controls were crossed to a motor neuron driver (D42-GAL4, UAS-MitoGFP) to label neuronal mitochondria. (A) Ventral nerve cord (VNC): Marf and Drp1 mutants exhibit clustered mitochondria in the soma. (B) Comparison of a proximal axonal segment in A3 and a distal segment in A5. Distal segments of A5 axons in Marf mutants contain many fewer mitochondria than proximal segments. (C) Marf mutants contain almost no mitochondria in boutons when co-stained with post-synaptic marker Discs Large 1 (Dlg1). Percentage of boutons with no mitochondria: Genomic rescue (0\%), Marf ${ }^{\text {B }}(89 \%)$, UAS-Marf (0\%), Drp12 (36\%) and Marf ${ }^{\mathrm{B}} ; \operatorname{Drp}^{12}$ (95\%).

DOI: 10.7554/eLife.03558.008

The following figure supplements are available for figure 3:

Figure supplement 1. Pre-synaptic, endocytic and postsynaptic markers are present in Marf mutant boutons. DOI: 10.7554/eLife.03558.009

Figure supplement 2. Mitochondrial trafficking defect in Marf mutants cannot be rescued by motor neuron expression of human MFN1 or MFN2

DOI: 10.7554/eLife.03558.010 
A

Low Frequency Stimulation $\left(0.2 \mathrm{~Hz}, 0.75 \mathrm{mM} \mathrm{Ca}^{2+}\right)$

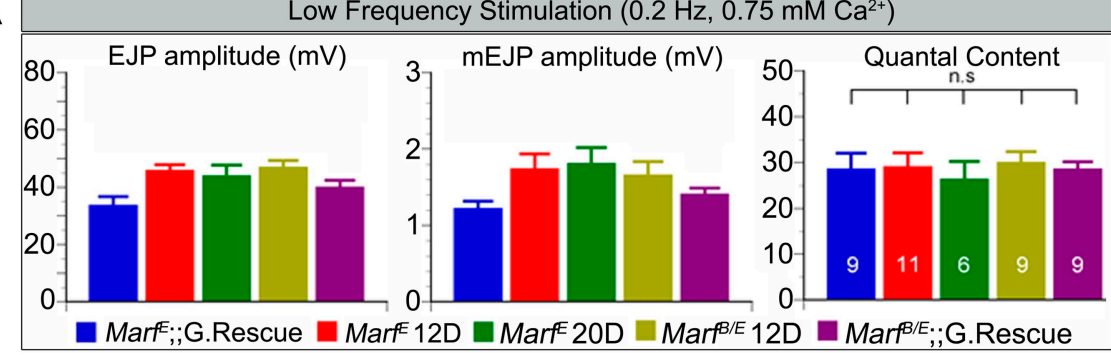

B

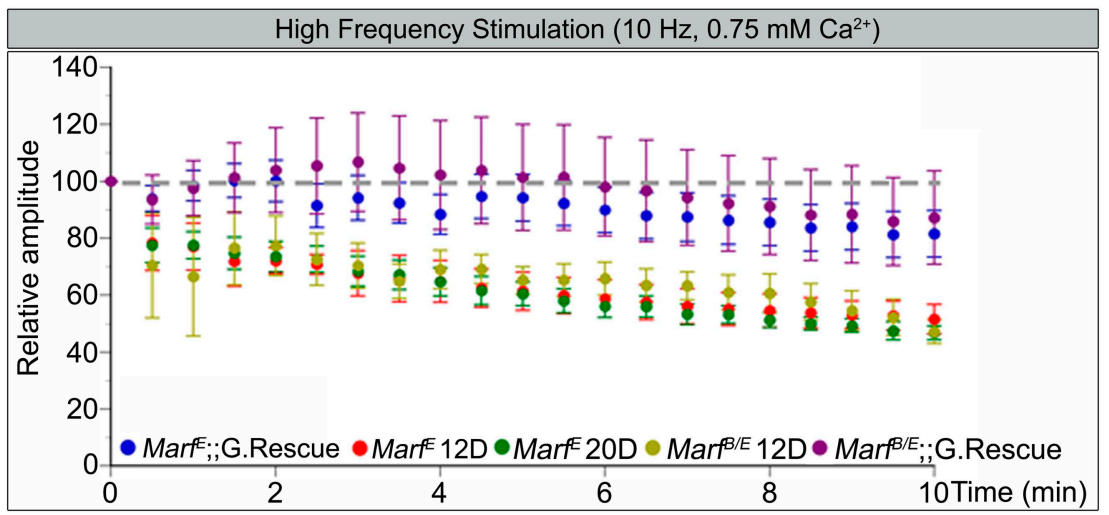

C

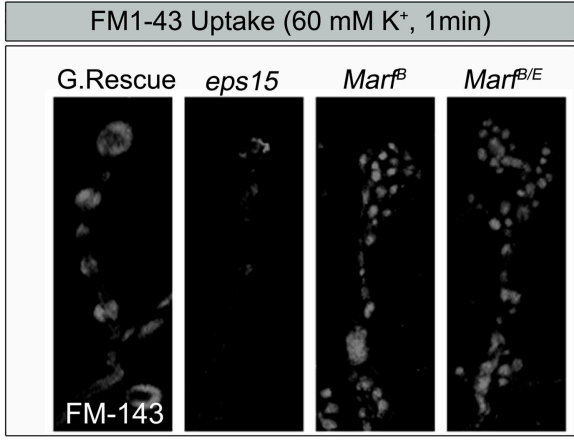

D

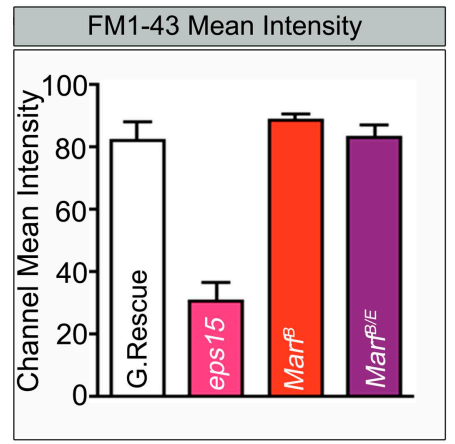

Figure 4. Marf is required to maintain synaptic transmission upon repetitive stimulation. (A) Excitatory Junctional Potentials (EJP) and miniature EJPs (mEJP) measured at $0.2 \mathrm{~Hz}$ in $0.75 \mathrm{mM} \mathrm{Ca}^{2+}$ are similar in Marf mutants (day 12 or day 20 old larvae) and controls. Hence, quantal content in Marf mutants is also similar to controls ( $n=6-11$ larvae assayed). (B) Controls display facilitation whereas Marf mutants (day 12 or day 20 old larvae) show a rundown at $10 \mathrm{~Hz}$ in $0.75 \mathrm{mM} \mathrm{Ca}^{2+}$. (C) Assessing endocytosis using FM-143 dye uptake at $60 \mathrm{mM}\left[\mathrm{K}^{+}\right]$for 1 min shows no obvious differences between wild type controls and Marf mutants. (D) Quantification of FM-143 uptake. Error bars represent \pm SEM. DOI: 10.7554/eLife.03558.011

mitochondria at synaptic terminals affect ATP levels required for vesicle mobilization at high frequency stimulation (Verstreken et al., 2005).

\section{Marf is required for proper NMJ development}

A striking difference between Marf mutants and Drp1 mutants is that loss of Marf severely affects NMJ morphology whereas loss of Drp1 does not affect NMJ development (Figure 3C, Figure 3-figure supplement 1 and Figure 2-source data 2). To visualize bouton morphology, we co-stained with Eps15, a presynaptic marker (Koh et alo, 2007) and Dlg1, a postsynaptic marker (Parnas et al., 2001). Marf mutant displayed a severe reduction in average bouton size (Figure 5A) accompanied by an increase in clustering and numbers of boutons when compared to controls (Figure $\mathbf{5 A}, \mathbf{C}$ ). This NMJ phenotype can be rescued by a Marf genomic rescue construct as well as ubiquitous expression of a Marf cDNA (Figure 5A,C). An increase in bouton number and reduction in size is also observed by ubiquitous knockdown of Marf using RNAi (Figure 5B,D and Figure 1-figure supplement 1C). 


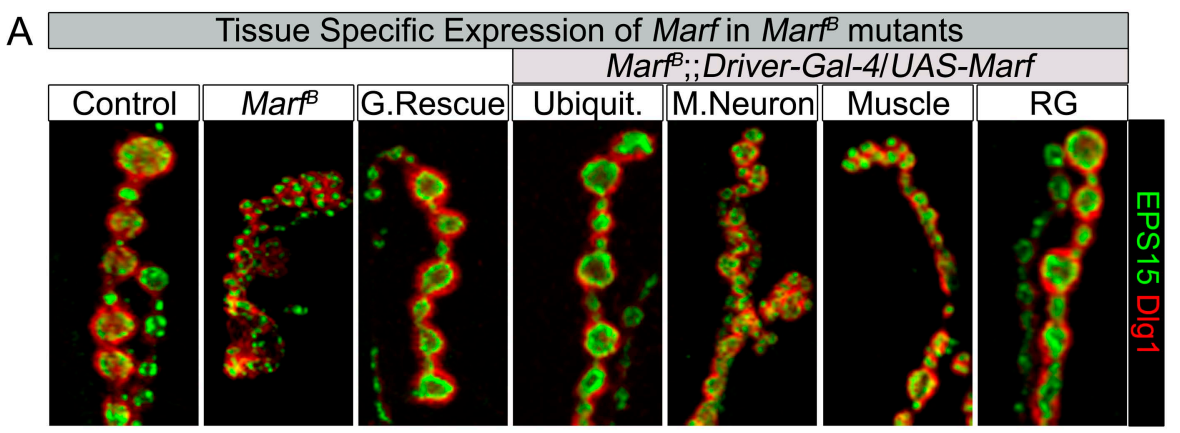

$\mathrm{B}$

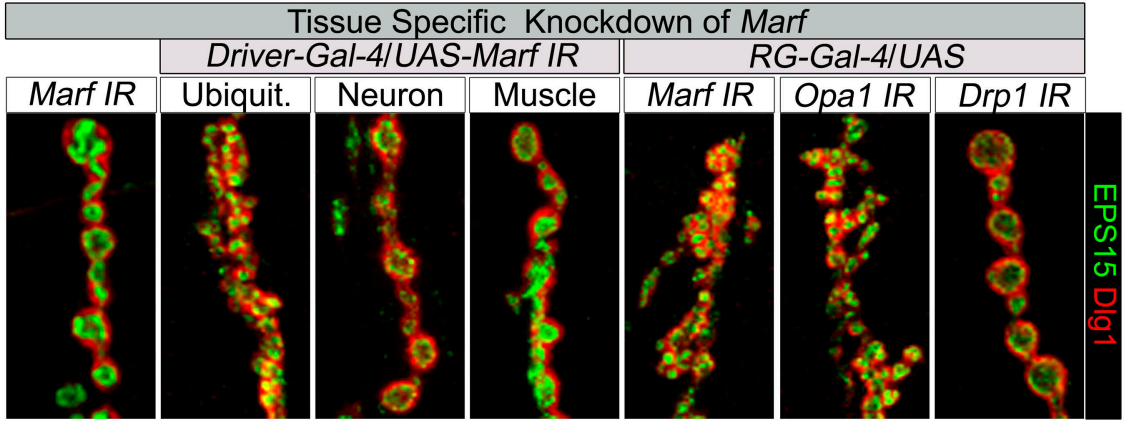

C Quantification of Boutons from (A) D Quantification of Boutons from (B)
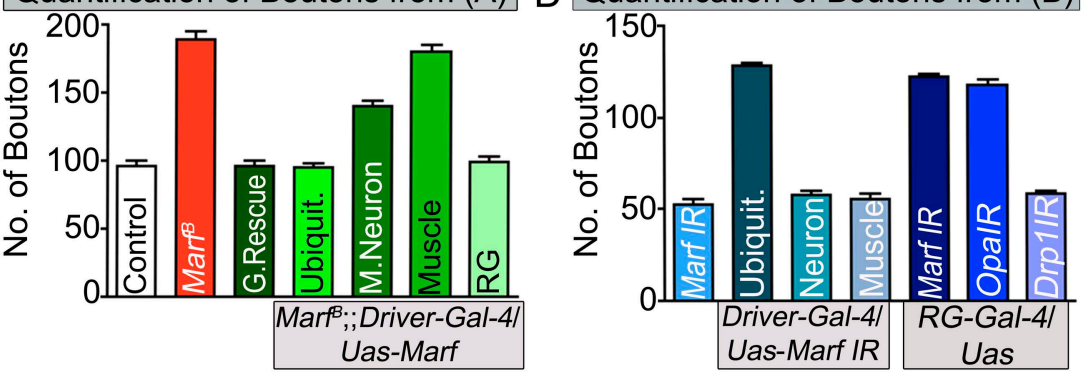

Figure 5. Loss of mitochondrial fusion but not fission in the ring gland results in altered bouton morphology. Third instar larvae NMJs from muscles 6/7 segments A3 were stained with pre-synaptic (EPS15) and post-synaptic (Dlg1) markers. (A) Ubiquitous (Tubulin-Ga/4) or ring gland (RG, Feb36-Gal4) expression of Marf rescue bouton morphology in Marf mutants, while motor neuron (D42-Gal4) or muscle (Mef-Ga/4) Marf expression did not. (B) Ubiquitous or RG specific knockdown of Marf or Opa1 (Poole et al., 2010) phenocopy the bouton phenotype in Marf mutants while knockdown of Drp1 (Drp1 IR knockdown of Drp1 mRNA is 82\% using ubiquitous driver Actin-Gal4) did not. (C and D) Quantification of bouton numbers from three independent experiments. Error bars represent \pm SEM. DOI: 10.7554/eLife.03558.012

The following source data and figure supplements are available for figure 5:

Source data 1. Tissue specific Gal4 screen to assess rescue of lethality and bouton morphology by Marf expression. DOI: 10.7554/eLife.03558.013

Source data 2. Tissue specific Gal4 screen to assess lethality and alterations to bouton morphology by Marf knockdown. DOI: 10.7554/eLife.03558.014

Figure supplement 1. Ring gland drivers tissues specificity.

DOI: 10.7554/eLife.03558.015

Since ubiquitous expression of the Marf cDNA rescues the NMJ morphology phenotype, we tested whether expression of Marf in MN, muscles or glial cells is able to rescue the phenotype. The NMJ phenotype is only partially rescued by Marf expression in MN (Figure 5A,C). Moreover, muscle, glial or MN and muscle expression of Marf does not alter the Marf mutant NMJ morphology (Figure 5A,C and Figure 5-source data 1). Consistent with these observations, RNAi knock down of Marf in MN, muscles, glia and MN and muscle does not affect bouton number or size at NMJs (Figure 5D and 
Figure 5-source data 2). This indicates that Marf expression is required in other cells than MN, muscles or glia.

\section{Mitochondrial fusion regulates NMJ morphology via a non-cell autonomous function in the ring glands}

To assess which other tissue/cells contribute to the NMJ defects in Marf mutants, we tested specific RNAi knockdown of Marf using Gal4 drivers that drive expression in different tissues including fat body, haemocytes, oenocytes, trachea or ring gland (RG) (Figure 5-source data 2). Knockdown of Marf with three independent RG-Gal4 drivers resulted in a NMJ phenotype similar to that observed in Marf mutants or ubiquitous knockdown of Marf (Figure 5B, Figure 5-source data 2 and Figure 5figure supplement 1), clearly showing a non-cell autonomous requirement for Marf in RGs. In addition, while knockdown of Marf in neurons and RG resulted in pupal lethality, only knockdown of Marf in RG significantly lengthened the third instar larva stage (8-10 days) (Figure 5-source data 2). Finally, expression of Marf in the RG using two different RG drivers rescued the bouton phenotype of Marf mutants (Figure 5A,C, Figure 5-source data 1 and Figure 5-figure supplement 1). Hence, Marf is required in RGs to regulate NMJ morphology in a cell non-autonomous manner.

Given that loss of Drp1 does not cause obvious developmental defects at NMJs (Figure 2-source data 2, Figure 3C and Figure 5B) (Drp1 IR knockdown of Drp1 mRNA is $82 \%$ using a ubiquitous driver Actin-Gal4), we tested whether loss of Opa1, another fusion protein (Cipolat et al., 2004; Chen et al., 2005), in RGs causes a bouton phenotype. A RG specific knockdown of Opa1 (Deng et al.,, 2008; Poole et al., 2010) causes a very similar alteration in synaptic morphology as Marf knockdown (Figure 5B). Moreover, Opa1 knockdown in RG also lengthens the larval stages and causes pupal lethality, similar to Marf knockdown (data not shown). Hence, both inner and outer mitochondrial fusion but not fission proteins alter bouton morphology and lengthen larval lifespan via RG, suggesting that the fusion proteins affect the same cell non-autonomous process.

RGs are responsible for production of hormones such as ecdysone (Huang et al., 2008) and juvenile hormone (Di Cara and King-Jones, 2013). These hormones regulate growth and differentiation of numerous tissues and control the proper timing of larval molts and metamorphosis (Yamanaka et al., 2012; Di Cara and King-Jones, 2013). Loss of production of ecdysone in RGs results in a lengthened larval stage ranging from 4 to 19 days (McBrayer et al., 2007, Talamillo et al., 2008; Rewitz et al., 2009). To determine if ecdysone production is affected we measured the levels of 20-hydroxyecdysone (20E) (Porcheron et al., 1976), in Marf mutants as well as animals with RG specific knockdown of Marf, Opa or Drp1. Marf mutants or knockdown of Marf and Opa1 in RG exhibit severely reduced levels of 20E when compared to control or knockdown of Drp1 in the RG or Drp1 mutant alleles (Figure 6A and Figure 2-source data 2). Restoring expression of Marf in the RGs of Marf mutants partially restores the 20E levels (Figure 6A). Moreover, the feeding of 20E to third instar larvae with RG specific knockdown of Marf rescued both the pupal lethality and NMJ morphology phenotype (Data not shown and Figure 6-figure supplement 1A). In summary, Marf and Opa1 but not Drp1 affect ecdysone production in the RG.

\section{Marf is required for lipid droplet formation in RG}

The production of ecdysone (steroid hormones) involves many steps following uptake of cholesterol. Drosophila lacks several biosynthetic enzymes for de novo cholesterol synthesis and depends on cholesterol uptake from the food (Clark and Block, 1959). In the RG, cholesterol is processed into 'free-cholesterol (FC)' in the ER (Miller, 2013). It is then transported into the mitochondrial inner matrix for processing by at least two cytochrome p450 enzymes (encoded by disembodied [Chavez et al., 2000] and shadow [Warren et al., 2002] in Drosophila) and finally secreted from the RG into the hemolymph (Gilbert, 2004). Because steroid hormones cannot be stored during Drosophila larva development, FC is stored in the form of cholesterol esters in lipid droplets (LDs) until there is a burst of ecdysone synthesis (Talamillo et al., 2008; Miller, 2013). This process of cholesterol ester storage and steroid synthesis is highly conserved from flies to mammals.

To assess cholesterol ester storage in LDs in RGs of wandering third instar larva, we first stained LDs with Nile Red, which marks neutral lipids that comprise LDs (Greenspan et al., 1985). This larval stage precedes the large burst of ecdysone that occurs at the larval-pupal transition (Yamanaka et al., 2012). Interestingly, the numbers of LDs are severely reduced in Marf mutants as well as in Marf knockdown in RGs (Figure 6B,C). Moreover, RG expression of Marf rescues the LD phenotype and even 

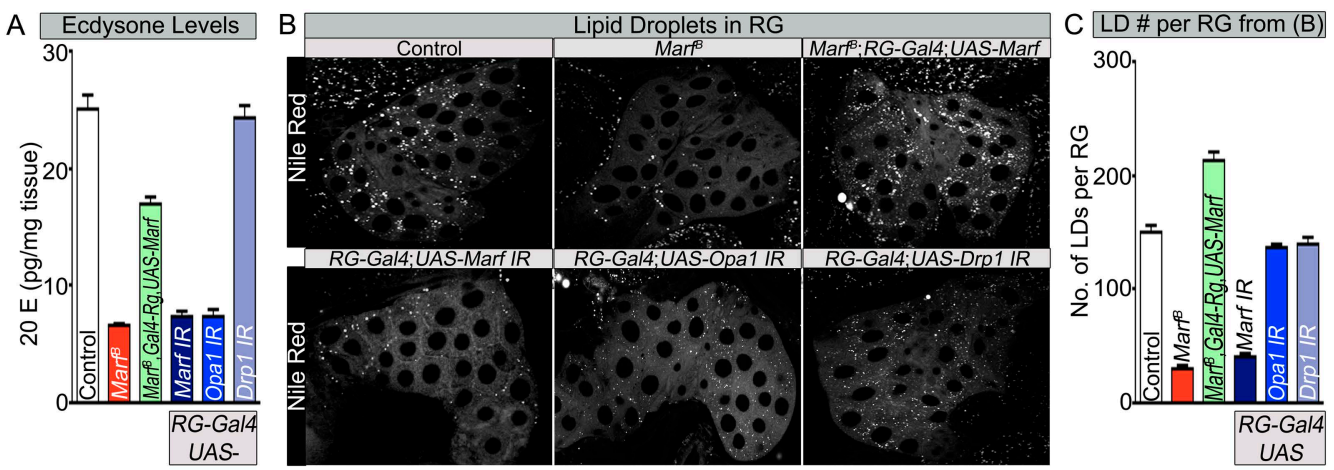

D

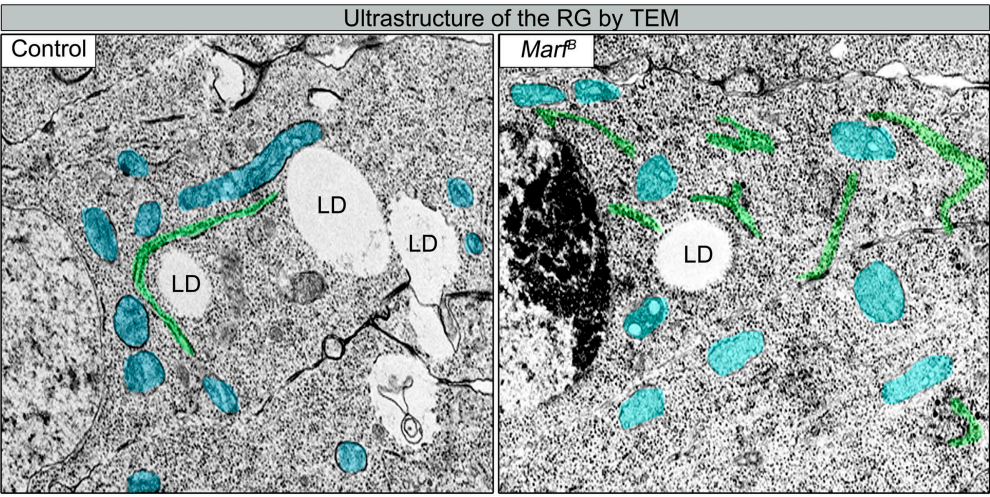

E Organelle Contacts

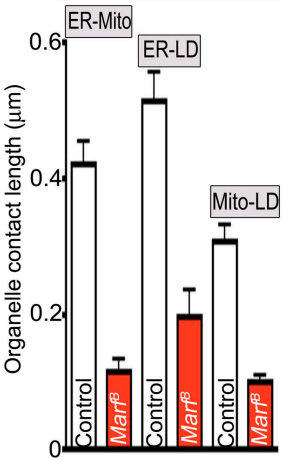

Figure 6. Both Marf and Opa1 regulate ecdysone synthesis in the ring gland, but only Marf promotes lipid droplet formation. (A) Both loss of Marf and Opa1 in the RG have reduced 20-hydroxyecdysone (20E) levels when compared to loss of Drp1 and controls. 20E levels are determined and normalized by weight. (B) Only loss of Marf in the RG results in reduced lipid droplets (LDs) when stained by Nile Red compared to loss of Opa1 or Drp1. (C) Quantification of $L D s$ in the ring gland (RG) from three independent experiments. (D) TEM sections of RG were the ER is labeled in green, mitochondria in blue and lipid droplets are labeled 'LD'. Marf mutants display increased ER fragmentation and reduced numbers of LDs when compared to Marf-genomic rescue control animals. (E) Marf mutants have reduced contact length between mitochondria and ER, ER and LD, and mitochondria and LD when compared to controls. Error bars represent \pm SEM.

DOI: 10.7554/eLife.03558.016

The following figure supplement is available for figure 6:

Figure supplement 1. Feeding of 20E rescues the NMJ morphology of RG specific knockdown of Marf. DOI: 10.7554/eLife.03558.017

increases the LDs numbers above control in Marf mutants, suggesting that Marf is necessary and sufficient for LD formation (Figure 6B,C). Interestingly, RG knockdown of Opa1 does not affect LD number (Figure $6 B, C$ ), suggesting that Marf and Opa1 have different roles in the RG. Our findings indicate that Marf plays a unique role in LD synthesis in RG and that it affects cholesterol ester storage. Loss of Opa1 on the other hand does not affect LD storage but like loss of Marf, impairs 20E production. Finally, loss of Drp1 or RG expression of Drp1 does not affect LD synthesis, nor does it affect 20E production (Figure 6A-C, Figure 2-source data 2 and Figure 6-figure supplement 1B,C). Taken together, the three mitochondrial GTPases have different roles in LD dynamics and ecdysone synthesis.

LD are generated from the ER through budding of the outer leaflet of the ER membrane (Walther and Farese, 2012). A physical link between the ER, LDs and mitochondria are often observed as these organelles collaborate to orchestrate numerous metabolic processes such as cholesterol transport and steroid synthesis (Issop et al., 2012; English and Voeltz, 2013). Indeed, human MFN2 has been shown to tether the mitochondria to the ER (de Brito and Scorrano, 2008). To assess the ultrastructural features of ER, LDs, and mitochondria in RGs, we performed TEM in RG. As shown in Figure 6D, Marf mutants exhibit a fragmented ER, reduced number of LD, and morphologically altered mitochondria when compared to controls. The contacts between the mitochondria and the ER, the ER and LD, as 
well as mitochondria and LD, are all severely reduced in Marf mutant RG (Figure 6D,E). This suggests that Marf promotes cholesterol ester storage in LDs possibly through inter-organelle connections.

\section{Marf integrates the functions of human MFN1 and MFN2}

Human MFN2 tethers mitochondria to the ER (de Brito and Scorrano, 2008) but this has not been documented for MFN1. Similarly, loss of MFN2 leads to ER stress (Ngoh et al., 2012; Sebastian et al., 2012; Munoz et al., 2013) but a role for MFN1 in ER function has not been reported. If Drosophila Marf mediates connections of mitochondria to ER and if this activity is required for ecdysone synthesis, expression of human MFN2 (Dorn et al., 2011) in the RG may rescue the loss of LDs, alleviate the bouton morphology defects and restore 20E levels in Marf mutants. We find that RG specific expression of human MFN2 restores the proper number of LD levels and organelle contacts in Marf mutants whereas expression of human MFN1 (Dorn et alo, 2011) does not (Figure 7A, $C$ and Figure 7-figure supplement 1), indicating that MFN2 specifically can rescue the defect in LD synthesis. However, RG expression of human MFN2 did not rescue the bouton phenotype of Marf mutants (Figure 7B,D). Moreover, ubiquitous expression of MFN1 or MFN2 alone (Daughterless-Gal4 and Tubulin-GAL4) does not rescue the lethality (Figure 1-figure supplement 1B), mitochondrial morphology (Figure 7figure supplement 2), mitochondrial trafficking to synapses (Figure 3-figure supplement 2), 20E levels, and the NMJ phenotypes (Figure 7), whereas ubiquitous co-expression of both MFN1 and MFN2 rescued all phenotypes (Figure 1-figure supplement 1B and Figure 7 ). These data indicate that MFN1 and MFN2 play non-redundant roles and have complementary functions that are integrated into a single protein in Drosophila Marf.

\section{Discussion}

How does loss of fission or fusion affect mitochondrial function? In the absence of fusion mixing of mitochondrial DNA and proteins may be severely impaired. Given that mitochondrial proteins are in an environment rich in oxygen radicals, lack of fusion may cause more damage than when fission is impaired (Chan, 2012). Simply stated, loss of fusion proteins like Marf, MFN1 or MFN2 may cause more severe phenotypes than the loss of a fission protein like Drp1. Moreover, proteins like Marf and Drp1 may perform other functions that are not directly related to fusion or fission, and hence affect other processes. Based on a careful phenotypic comparison of loss of Marf and Drp1 in Drosophila we find many similarities and differences.

Marf mutants display small mitochondria whereas Drp1 mutants exhibit large fused mitochondria. Interestingly, both mutants accumulate mitochondria in the cell body of the neurons and the proximal axonal segments (Figure 3A). In Drp1 mutants, the mitochondria seem to be severely elongated in axons where they fail to reach the NMJs, as previously described (Verstreken et al., 2005). The impairment in axonal transport is thought to be due to the fact that the mitochondria are hyperfused and cannot easily be transported. Indeed, loss of Marf in Drp1 mutants can restore mitochondrial trafficking proximally but distal axonal trafficking is still impaired (Figure 3B). In Marf mutants, even though mitochondria are small and can enter the axons, the numbers of mitochondria that travel distally toward the NMJs are dramatically reduced (Figure 3B). Hence, loss of Marf impairs mitochondrial trafficking and longer axons are more severely affected than shorter axons. Since longer axons are more severely affected in CMT2A patients (Scherer, 2011), defects in mitochondrial trafficking may be at the root of some of the phenotypes associated with the disease.

Mfn2 has been implicated in axonal transport via binding to Miro2. Indeed, knockdown of MIRO2 in cultured vertebrate neurons affects mitochondrial transport in an identical fashion as loss of MFN2 (Misko et al., 2010). However, the severity of mitochondrial transport that we observe in Marf mutants is much less pronounced than what has been described in dmiro mutants (Guo et al., 2005) and what we observe when dmiro is lost. Moreover, removal of dmiro in Marf mutants dramatically enhances the Marf phenotype and almost abolishes axonal localization of mitochondria (Figure 3-figure supplement 2), arguing that Marf cannot be solely responsible for mitochondrial transport in Drosophila.

A comparison of the presence of mitochondria at NMJ synapses shows that Marf mutants have fewer mitochondria than Drp1 mutants (Figure 3C). Moreover, Marf mutants but not Drp1 mutants display a severe increase in small clustered boutons (Figure 2-source data 2, Figures 3C and 5). The small and clustered boutons have also been observed in other mutants like endophilin (Dickman et al., 2006), synaptojanin (Dickman et al., 2006), eps15 (Koh et al., 2007), dap 160 (Koh et al., 2004), flower (Yao et al., 2009) and dmiro (Guo et al., 2005). However, unlike in Marf mutants, the bouton 


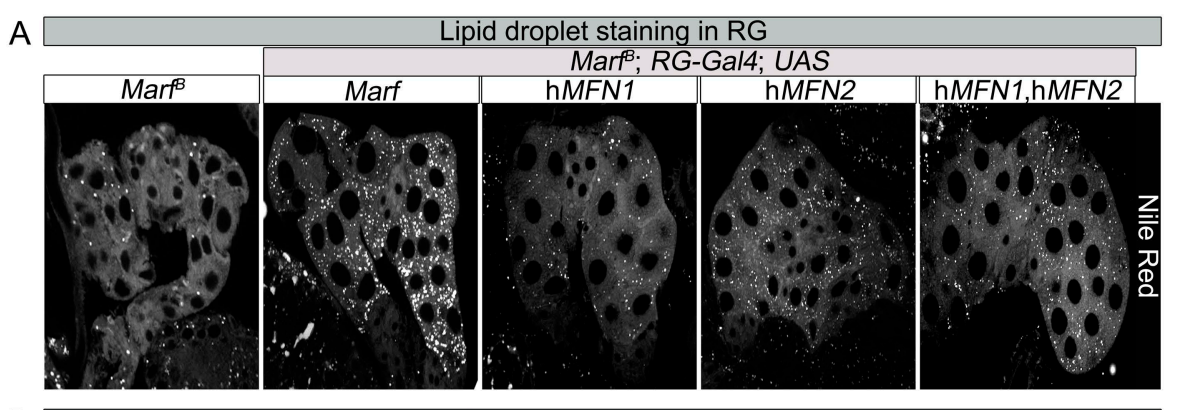

B
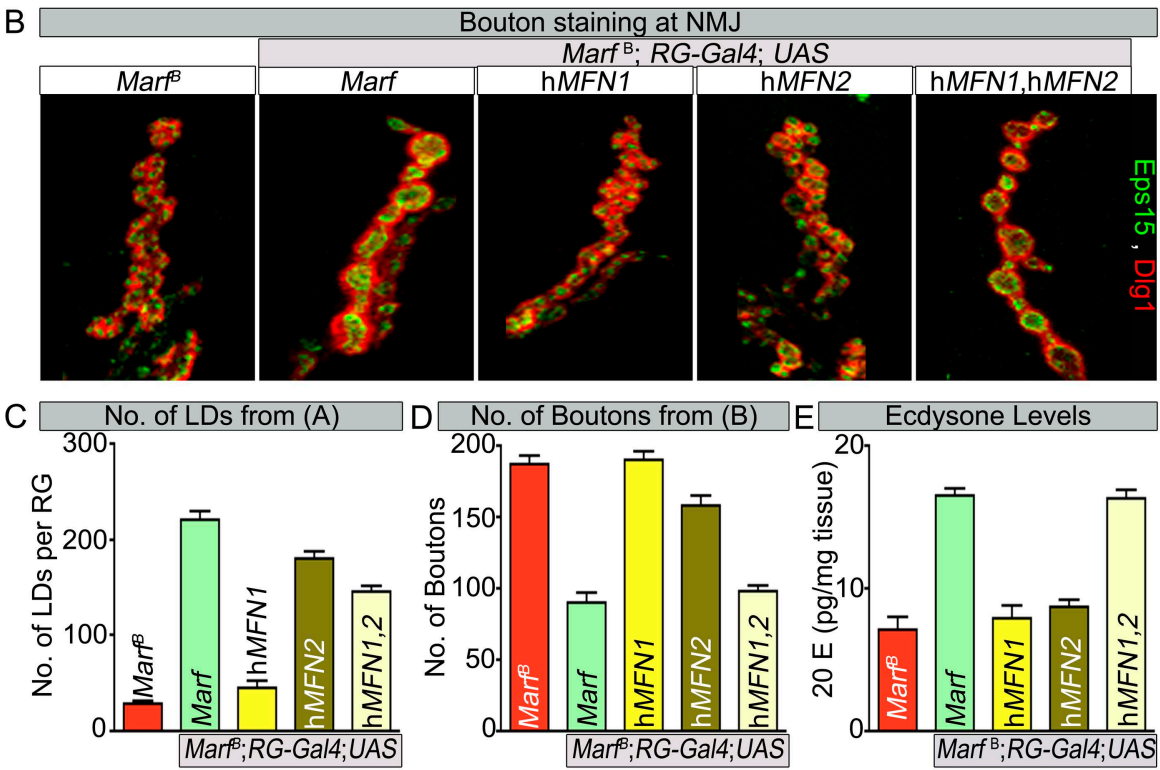

Figure 7. Human MFN2 restores LD numbers but both human MFN1 and MFN2 are required for steroid-hormone production in the ring glands. (A) Rescue of lipid droplets numbers stained by Nile Red in Marf ring glands (RG) by MFN2 and MFN1/MFN2 co-expression, but not MFN1. (B) Rescue of Marf bouton morphology by expressing MFN1/MFN2 in RGs (Feb36-Gal4). Expression of MFN1 or MFN2 alone does not rescue the phenotype. (C-E) Quantification in control and Marf mutants for: (C) LDs (D) Boutons and (E) Ecdysone (20E levels) as described in Figures 5 and 6. Error bars represent \pm SEM.

DOI: 10.7554/eLife.03558.018

The following figure supplements are available for figure 7 :

Figure supplement 1. RG expression of human MFN2 restores organelle contact lengths in Marf mutants. DOI: 10.7554/eLife.03558.019

Figure supplement 2. Muscle expression of either human MFN2 or MFN1 does not fully restores mitochondrial morphology in Marf mutants.

DOI: 10.7554/eLife.03558.020

phenotypes are fully rescued by neuronal expression of the cognate protein within MN in the above mentioned mutants. Moreover, knockdown of Marf in neuron, muscle or glia does not recapitulate the bouton phenotype observe in Marf mutants (Figure 5B and Figure 5-source data 2), suggesting a unique cell non-autonomous requirement of Marf for proper NMJ morphology.

Marf mutants exhibit two obvious phenotypes at NMJs: a severe depletion of mitochondria and a doubling of the number of boutons combined with a severe reduction in size whereas Drp1 mutants only exhibit a severe reduction in mitochondria. However, our electrophysiological studies show that loss of Marf does not affect basal synaptic transmission (Figure 4) similar to what is observed in Drp1 mutants (Verstreken et al., 2005). Both respond similarly to wild type NMJs when stimulated at 0.2 $\mathrm{Hz}$ and both show a progressive run down at $10 \mathrm{~Hz}$ when compared to controls. Moreover, endocytosis using FM1-43 and $60 \mathrm{mM} \mathrm{K}^{+}$is not impaired in Marf and Drp1 mutants, suggesting a defect in reserve 
pool mobilization in both mutants (Verstreken et al., 2005, 2008). The data also show that the bouton defects observed in Marf mutants do not contribute to the run down in synaptic transmission since Drp 1 boutons are normal in number and size yet also have a run down in synaptic transmission (Figure 2-source data 2, Figures 3 and 4; [Verstreken et al., 2005]).

Loss of Marf in RG recapitulates the bouton phenotype observed in Marf mutants and expression of Marf in RG fully rescues this phenotype (Figure 5 and Figure 5-source data 1). Interestingly, both Marf and Opa1 are required for steroid hormone production and both lead to extended larval lifespan when knocked down in the RG only (8-10 days), whereas Drp1 mutations do not affect steroid hormone synthesis. Reduction of ecdysone production by knockdown of the prothoracicotropic hormone receptor (torso) in the RG also leads to an extended larval lifespan (9 days) (Rewitz et al., 2009) and an increased growth of NMJs (Miller et al., 2012). Interestingly, knockdown of Drosophila SUMO (dsmt3) in RG lead to a defect in cholesterol import in the RG, reduced $20 \mathrm{E}$ levels and an extended larval lifespan (19 days) (Talamillo et al., 2008). Hence, the severe reduction in ecdysone synthesis in Marf mutant RG underlies the prolonged larva stages and NMJ morphological defects.

The reduction in the number of LDs in RGs when Marf is lost suggests that these RGs are unable to store cholesterol (Figure 6B,C). This storage of cholesterol esters probably permits the RG to produce large amounts of ecdysone when needed, especially at the larval stage and larval to pupal transitions. Cholesterol storage and steroid hormone biosynthesis requires both the ER and mitochondria in vertebrates (Miller, 2013) but loss of MFN1 or MFN2 have not been shown to affect LD synthesis. Defects of anchoring mitochondria to the ER and LDs in Marf RGs argue that these defects lead to the loss of LD and production of ecdysone (Figure 6). In agreement with this hypothesis, expression of human MFN2, which tethers ER to mitochondria (de Brito and Scorrano, 2008), in Marf mutants restores LD synthesis and organelle contacts (Figure 7A, Figure 7C and Figure 7-figure supplement 1). Moreover, expression of human MFN2 in RNAi mediated Marf knockdown in neurons and muscles rescues ER morphology and stress (Debattisti et al., 2014). However, MFN2 expression alone in Marf mutant RG did not restore ecydsone synthesis (Figure 7E), arguing that there are other mitochondrial defects associated with the loss of Marf (Figure 8).

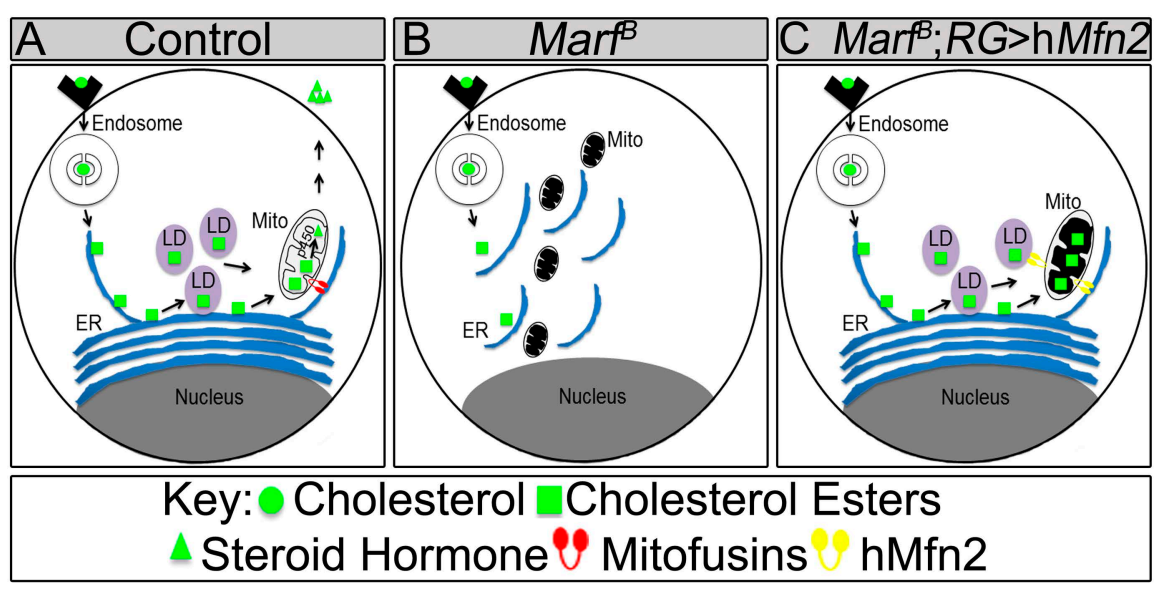

Figure 8. Model of Marf dual function in steroid synthesis in the ring glands. (A) In wild type ring glands (RG), cholesterol must enter the cell first. Then, cholesterol undergoes a series of modifications in endosomes and along the ER to become free-cholesterol. Then, free-cholesterol is transferred into the mitochondrial inner matrix, where it is processed from free-cholesterol to steroid hormone by p450 enzymes. The steroid hormone is then secreted. As Drosophila larva develops it stores cholesterol in the form of cholesterol ester in lipid droplets (LDs) in order to accumulate a reserve of substrate so it can generate bursts of steroid hormone when needed. These LDs require the ER for synthesis. (B) In Marf mutants, the ER is fragmented and LD formation is severely reduced. (C) RG-specific expression of MFN2 in Marf mutant restores LD numbers but does not rescue hormone synthesis, suggesting that Marf has a second function within the mitochondria.

DOI: 10.7554/eLife.03558.021 
Our data show that co-expression of human MFN1 and MFN2 fully rescue the observed phenotypes in Marf mutants (Figure 7). Although RG-specific expression of MFN1 in Marf mutants did not restore LD numbers or organelle contacts (Figure 7-figure supplement 1), MFN1 is still necessary for ecdysone synthesis together with MFN2, suggesting a role downstream of cholesterol ester storage for both proteins (Figure 8). Moreover, knockdown of Opa1 in RG did not alter LD numbers but causes reduced 20E levels and aberrant NMJs (Figure 6). Opa1 resides within the inner mitochondrial membrane, suggesting its role in ecdysone synthesis is within the mitochondria. Ecdysone synthesis within the mitochondria requires two cytochrome p450 enzymes encoded by disembodied (Chavez et al., 2000) and shadow (Warren et al., 2002). Hence, it is likely that impairment in fusion but not fission affects the function of these enzymes (Figure 8).

Opa1 and MFN2 but not Drp1 have been implicated in vertebrate steroidogenesis (Issop et alo, 2012). Interestingly, in placental trophoblast cells (BeWO) in culture the loss of OPA-1 promotes progesterone production by $70 \%$ whereas loss of MFN2 has been reported to lead to a $20 \%$ decrease in progesterone production (Wasilewski et al., 2012). In contrast, testosterone production in MA-10 Leydig cells was unaffected by loss of OPA1 (Rone et al., 2012) whereas loss of MFN2 did affect testosterone production by $40 \%$ in MA-10 Leydig cells (Duarte et al., 2012). Hence, in both vertebrate endocrine cells, loss of MFN2 or OPA-1 affected steroids very differently as we observe very similar phenotypes associated with the loss of either protein. Our study also suggests that MFN2 functions upstream of cholesterol entry into the mitochondria at the cholesterol storage stage, since MFN2 restores LD synthesis in Drosophila RG. However, rescuing LD production is not sufficient to restore ecdysone synthesis, suggesting a secondary defect (Figure $8 \mathrm{C}$ ). In summary, our data indicate that MFN1 and MFN2 have separate functions in vivo that are integrated in a single protein in fly Marf.

\section{Materials and methods}

\section{Fly Strains, maintenance of flies and larvae}

Flies were obtained from the Bloomington Drosophila Stock Center at Indiana University (BDSC) unless otherwise noted. All flies were kept in standard media and stocks were maintained at room temperature $\left(21-23^{\circ} \mathrm{C}\right)$. For all the larvae experiments described, flies were allowed to lay embryos for $48 \mathrm{hr}$ on grape juice plates with yeast paste. Hemizygous mutant larvae and wild type controls were isolated via GFP selection at the first instar phase and transferred to standard fly food for the duration of their development.

The following stocks were used in this study:

1. $y^{1} w^{*} P\{$ neoFRT $\} 19 A$

2. $y^{1} w^{\star}$ Marf $A, B, C, E, F, G$ or $H$ P $\left\{\right.$ neoFRT\}19A/FM7c,Kr-Gal4 UAS-GFP, sn ${ }^{+}$

3. yw eyFLP GMR-LacZ; $y^{+}$; Drp12 FRT4OA/CyO, Kr-Gal4 UAS-GFP

4. $c l(1) P\{$ neoFRT $\} 19 A / D p(1 ; Y) y+v+$ ey-FLP

5. $y^{1} w^{118}$ ey-FLP; Drp $1^{2}$ FRT4OA/CyO, Kr-Gal4 UAS-GFP

6. $y^{1} w^{*}$ MarfB or E P\{neoFRT\}19A/FM7c,Kr-Gal4 UAS-GFP;; Genomic Marf-HA/TM6B,Tb+

7. $y^{1} w^{*}$ Marf ${ }^{B}$ P neoFRT\}19A/FM7c,Kr-Gal4 UAS-GFP;; UAS-MarfHA/TM6B,Tb

8. $y$ w; D42-Gal4, UAS-mito-HA-GFP, e/TM6B,Tb

9. y w; Drp $1^{2}$ FRT4OA/CyO, Kr-Gal4 UAS-GFP; D42-Gal4, UAS-mito-HA-GFP, e/TM6B,Tb

10. $y^{1} w^{\star}$ Marf ${ }^{B} P\left\{\right.$ neoFRT $19 A / F M 7 c, K r-G a l 4$ UAS-GFP; Drp $1^{2}$ FRT40A/CyO, Kr-Gal4 UAS-GFP

11. y w; Df(2L)burK1, eps15[e75]/Cyo; twi-Gal4 UAS-2xEGFP

12. $y^{1} w^{*}$ Marf ${ }^{B} P\{$ neoFRT $\} 19 A / F M 7 c, K r-G a l 4$ UAS-GFP;; Tub-Gal4/TM6B,Tb

13. $y^{1} w^{*}$ Marf ${ }^{B} P\{$ neoFRT $19 A / F M 7 c, K r-G a l 4$ UAS-GFP; DA-Gal4

14. $y^{1} w^{*}$ Marf ${ }^{B}$ PneoFRT\}19A/FM7c,Kr-Gal4 UAS-GFP;; Mef-Gal4/TM6B,Tb

15. $y^{1} w^{\star}$ Marf $P\{$ neoFRT\}19A/FM7c,Kr-Gal4 UAS-GFP; Feb36-Gal4/CyO, Kr-Gal4 UAS-GFP

16. $y^{1} w^{*}$ Marf ${ }^{B}$ P\{neoFRT\}19A/FM7c,Kr-Gal4 UAS-GFP;; Mai60-Gal4/TM6B,Tb

17. $y$ w; ; UAS-Marf IR/T(2;3)TSTL,Cyo:TM6b, Tb

18. $y w_{; ;}$UAS-Drp1 IR/T(2;3)TSTL,Cyo:TM6b, Tb

19. $y$ w; ; UAS-dmiro IR/T(2;3)TSTL,Cyo:TM6b, Tb

20. $y^{1} w^{\star}$ Marf ${ }^{B} P\{$ neoFRT $19 A / F M 7 c, K r-G a l 4$ UAS-GFP;; UAS-MFN1/TM6B,Tb

21. $y^{1} w^{\star}$ Marf alleles P\{neoFRT\}19A/FM7c,Kr-Gal4 UAS-GFP;; UAS-MFN2/TM6B,Tb

22. yw eyFLP GMR-LacZ; $y^{+}$; Drp $1^{1}$ FRT4OA/CyO, Kr-Gal4 UAS-GFP

23. Drpi ${ }^{[T 26]} \mathrm{cn}$ bw sp/CyO, Kr-Gal4 UAS-GFP 
24. $y ; \operatorname{Drp}]^{[K G 03815] / C y O ; r y}$

25. w; UAS-Drp1/TM6C, Sb Tb

26. Gal4 BDSC fly lines listed on Figure 5-figure supplement 1

\section{Screen and mapping of Marf}

$y, w, P\left\{\right.$ neoFRT $19 A^{\text {isogenized }}$ (iso) male flies were treated with low concentration of ethylmethanesulfonate to induce mutations, and mutant alleles which showed ERG defects were isolated as described (Xiong et al., 2012; Zhang et al., 2013; Yamamoto et al., 2014).

For mapping of the Marf group, male large duplications ( 1-2 Mb) covering the $\mathrm{X}$ chromosome (Haelterman et al., 2014) were crossed with female $y, w$ mut ${ }^{\star}, P\{$ neoFRT $\} 19 A^{\text {isogenized }}$ flies that were balanced with FM7c,Kr-GAL4,UAS-GFP(Kr > GFP). For the Marf group, the lethality of all alleles were rescued by $D p(1 ; Y) d x[+] 5, y[+] / C(1) M 5(4 C 11 ; 6 D 8+1 A 1 ; 1 B 4)$. Marf alleles complemented with all the available deficiencies covered by $D p(1 ; Y) d x[+] 5, y[+] / C(1) M 5$ except Df(1)Exel6239 (Parks et al., 2004; Cook et al., 2012). We then performed Sanger sequencing for genes located to this region and identified mutations in Marf.

\section{Marf genomic and cDNA constructs}

A $6.1 \mathrm{~kb}$ genomic rescue fragment (X: 6259600...6265700, Drosophila melanogaster Release 5.7) was amplified using PCR from the P[acman] CH322-102K19 (Venken et al., 2009). This DNA fragment was then subcloned into the HindIII and Kpnl sites of the P element transformation vector $P\{C a S p e R-4-H A\}$ (Yao et al., 2009) and sequenced. For cDNA constructs, the CDS of Marf was retrieved from cDNA clones RE04414 (Stapleton et al., 2002), respectively, and subcloned into pUAST-HA vector (Ohyama et al., 2007) using Notl and Xbal sites. Cloning and DNA purification were performed based on standard protocols. All constructs were sequenced before injection.

\section{Generation of transgenic miRNAi lines for Drosophila Marf, Drp1 and dmiro}

As previously described in Yao et al. (2008), we chose the 22 nucleotides of the coding sequence of Marf, Drp1, or dmiro as target sequences listed in lowercase and bold in the sequences shown below. In oligo-1, the third nucleotide from 3' end was changed to $C$. To synthesize essential backbone for miRNAi production, four long primers were designed. The first PCR product was generated by oligo-1 and -2. With the first PCR template, the final construct was generated by using common oligo-3 and -4 then digested with EcoRI and Notl and cloned into the pUAST transformation vector.

Marf-oligo-1

GGCAGCTTACTTAAACTTAATCACAGCCTTAATGTtaaatgtggtgaacatcaaCca TAAGTTAATATACCATATC

Marf-oligo2

AATAATGATGTTAGGCACTTTAGGTACtaaatgtggtgaacatcaaacaTAGATATGGTATATTAACTTATGGT

Drp1-oligo1

GGCAGCTTACTTAAACTTAATCACAGCCTTTAATGTcaacgcacgtggtcaacctCacTAAGTTAATAT ACCATATC

Drp1-oligo2

AATAATGATGTTAGGCACTTTAGGTACcaacgcacgtggtcaacctaacTAGATATGGTATATTAACTTAGTGA

Miro-oligo1

GGCAGCTTACTTAAACTTAATCACAGCCTTTAATGTgaatgtggttaattg catcCacTAAGTTAATATA CCATATC

Miro-oligo2

AATAATGATGTTAGGCACTTTAGGTACgaatgtggttaattgcatcaacTAGATATGGTATATTAACTTAGTGG

Common oligos

Oligo-3

GGCGAATTCATGTTTAAAGTCCACAACTCATCAAGGAAAATGAAAGTCAAAGTTGGCAGCTTACTT AAACTTAATCA 


\section{Electroretinograms}

For ERG recording, $y w{ }^{*}$ mut (lethal) FRT19A/FM7c, Kr-Gal4, UAS-GFP flies were crossed to $y w P\{w+\}$ cl(1) FRT19A/Dp(1;Y)y+; eyFLP or y w; Drp $1^{2}$ FRT40A/CyO crossed to y w, eyFLP; Drp $1^{2}$ FRT40A/CyO to generate flies with mutant clones in the eyes and ERGs were performed as previously described (Ly et al., 2008). Briefly, adult flies were glued to glass slides. A recording probe was placed on the surface of the eye, and a reference probe was inserted in the thorax. A 1-s flash of white light was given, and the response was recorded and analyzed by the AXON ${ }^{\mathrm{TM}}$-pCLAMP8 software.

\section{Transmission electron microscopy (TEM) of laminas and ring glands}

TEM of photoreceptor terminals (Verstreken et al., 2003) and ring glands (Bellen and Budnik, 2000) was performed as described. TEM of photoreceptor terminals and ring glands were done using a Ted Pella Bio Wave processing microwave with vacuum attachments. Briefly, fly heads or third instar larva were dissected and fixed at $4{ }^{\circ} \mathrm{C}$ in $4 \%$ paraformaldehyde, $2 \%$ glutaraldehyde, $0.1 \mathrm{M}$ sodium cacodylate, and $0.005 \% \mathrm{CaCl}_{2}\left(\mathrm{PH}\right.$ 7.2) overnight, post-fixed in $1 \% \mathrm{OsO}_{4}$, dehydrated in ethanol and propylene oxide, and then embedded in Embed-812 resin (Electron Microscopy Sciences, Hatfield, PA). Photoreceptors or ring glands were then sectioned and stained in $4 \%$ uranyl acetate and $2.5 \%$ lead nitrate. TEM images of PR sections were taken using a JEOL JEM 1010 transmission electron microscope with an AMT XR-16 mid-mount 16 mega-pixel digital camera.

\section{Mitochondria functional assays for Marf and Drp1 mutants}

Staining of mitochondria membrane potential (MMP) by Tetramethylrhodamine ethyl ester (TMRE; Molecular Probes, Life Technologies, Grand Island, NY) and ROS by dihydroethidium dye (DHE; Sigma, St. Louis, MO) in live muscles, larvae were prepared and stained as described in Shidara and Hollenbeck (2010). Live images were acquired using a 40x water immersion lens and a Zeiss LSM510 confocal microscope. ATP levels in larvae was determined as described (Park et al., 2006) using a kit (Invitrogen, Life Technologies, Grand Island, NY). Quantification of ETC enzymatic activity assay and aconitase assay were performed on isolated mitochondria extracted as previously described (Graham et al., 2010; Zhang et al., 2013). Enzymatic activity assays were performed as previously described (Emptage et al., 1983; Das et al., 2001; Graham et al., 2010; Zhang et al., 2013). Aconitase activity assays were performed as previously described in Graham et al. (2010); Zhang et al. (2013).

\section{Dissection, immunostaining and lipid droplet staining by Nile Red}

For muscle or NMJ immunostaining, dissection and immunostaining of third instar larvae were performed as described in Bellen and Budnik (2000). Briefly, third instar larvae were fixed in $3.7 \%$ formaldehyde for $20 \mathrm{~min}$ at room temperature and washed in $0.4 \%$ Triton X-100. Primary antibodies were used at the following dilutions: mouse anti- ATP5A 1:500 (Abcam, Cambridge, MA), chicken anti-GFP 1:1000 (Abcam, Cambridge, MA), mouse anti-DLG 1:250 (DSHB, [Parnas et al., 2001]), guinea pig anti-EPS15 1:2000 (Koh et al., 2007), mouse anti-BRP 1:1000 (Wagh et al., 2006), rabbit anti- $\alpha$-adaptin 1:500 (Gonzalez-Gaitan and Jackle, 1997), mouse anti-Glutamate receptor Ila (DSHB, lowa City, IA, [Schuster et al., 1991]), guinea pig anti-Dap160 1:500 (Roos and Kelly, 1998), rabbit anti-HRP 1:1500 (Jackson ImmunoResearch, West Grove, PA), guinea pig anti-endophilin 1:200 (Verstreken et al., 2002), rabbit anti-synaptojanin (Verstreken et al., 2003), and rabbit anti-Drosophila vesicular glutamate transporter (DVGlut) 1:2000 (Daniels et al., 2004). Alexa 488 conjugated (Invitrogen), and Cy3 or Cy5 conjugated secondary antibodies (Jackson ImmunoResearch, West Grove, PA) were used at 1:250. Samples were mounted in VECTASHIELD (Vector Labs, Burlingame, CA).

For Lipid Droplet staining, third instar larvae were dissected in cold PBS and fixed in 4\% paraformaldehyde for $30 \mathrm{~min}$. Larvae were rinsed several times with $1 \times$ PBS to remove fixative and incubated for $10 \mathrm{~min}$ at 1:1000 dilution of PBS with $1 \mathrm{mg} / \mathrm{ml}$ Nile Red (Sigma, St. Louis, MO). Subsequently the tissues were rinsed with PBS and immediately covered with VECTASHIELD (Vector Labs, Burlingame, CA) for same-day imaging. 
All confocal figures were acquired with confocal microscope (LSM510; Zeiss) using Plan Apochromat $40 \times$ NA 1.4 and Plan Apochromat $63 \times$ NA 1.4 objectives (Zeiss), followed by processing in LSM software (Zeiss), ImageJ, and Photoshop (Adobe).

\section{Electrophysiology and FM-143 labeling}

Larval electrophysiological recordings were performed as described in Koh et al. (2004). For labeling the exo-endo cycling pool (ECP) of vesicles, FM1-43 assays were performed as described (Verstreken et al., 2005, 2008). Live images were acquired using a 40x water immersion lens and a Zeiss LSM510 confocal microscope.

\section{Ecdysteroid (20E) titers}

Ecdysteroid levels were quantified by ELISA following the procedure described by Porcheron et al. (1976), and adapted by Pascual et al. (1995). For sample preparation, 20 to 30 staged larvae were weighed and preserved in $600 \mu \mathrm{l}$ of methanol. Prior to the assay, samples were homogenized and centrifuged $(10 \mathrm{~min}$ at $18,000 \times \mathrm{g})$ twice and the resultant methanol supernatants were combined and dried. Samples were resuspended in $50 \mu \mathrm{l}$ of enzyme immunoassay (EIA) buffer $(0.4 \mathrm{M} \mathrm{NaCl}, 1 \mathrm{mM}$ EDTA, 0.1\% BSA in 0.1 M phosphate buffer). 20E (Sigma, St. Louis, MO) and 20E-acetylcholinesterase (Cayman Chemical, Ann Arbor, MI) were used as the standard and enzymatic tracer. Absorbance was read at $450 \mathrm{~nm}$ using a FLUOstar Optima Spectrophotometer (BMG Labtech), results are expressed as 20 E equivalents.

\section{Acknowledgements}

We thank the Bloomington Drosophila Stock Center for flies and the Developmental Studies Hybridoma Bank for antibodies. We are grateful to Dr GW Dorn (UAS-MFN1 and UAS-MFN2), Dr AJ Whitworth (Marf antibody) and Dr M Guo (UAS-Opa IR and UAS-Drp1) for fly stocks and antibodies. We thank Drs MF Wangler, ES Seto, KL Schulze and K Vankatachalam for critical reading. We thank $\mathrm{H}$ Pan and Y He for injections. We thank Dr S Jaiswal with help with qPCR of DRP1. Confocal microscopy at BCM is supported by the Intellectual and Developmental Disabilities Research Center (NIH 5P30HD024064). HS was supported by NIH 5R01GM067858, NIH T32 NS043124-11 and the Research Education and Career Horizon Institutional Research and Academic Career Development Award Fellowship 5K12GM084897. VB was supported by the NIH (5T32HD055200) and the Edward and Josephine Hudson Scholarship Fund. BX was supported by the Houston Laboratory and Population Science Training Program in Gene-Environment Interaction from the Burroughs Wellcome Fund (Grant No. 1008200). W-LC was supported by Taiwan Merit Scholarships Program sponsored by the National Science Council (NSC-095-SAF-I-564-015-TMS). SY was supported by a fellowship from the Nakajima Foundation and the Jan and Dan Duncan Neurological Research Institute at Texas Children's Hospital. This project was funded in part by NIH RC4GM096355-01 and gifts from the Robert A and Renee E Belfer Family Foundation, the Huffington Foundation, and Target ALS. HJB is an Investigator of the Howard Hughes Medical Institute.

\section{Additional information}

Funding

\begin{tabular}{|c|c|c|}
\hline Funder & Grant reference number & Author \\
\hline National Institutes of Health & RC4GM09355-01 & Hugo J Bellen \\
\hline $\begin{array}{l}\text { Belfer Center for Science and } \\
\text { International Affairs, Harvard } \\
\text { University }\end{array}$ & & Hugo J Bellen \\
\hline Huffington Foundation & & Hugo J Bellen \\
\hline Project A.L.S & Target ALS & Hugo J Bellen \\
\hline $\begin{array}{l}\text { Howard Hughes Medical } \\
\text { Institute }\end{array}$ & & Hugo J Bellen \\
\hline National Institutes of Health & 5R01GM067858 & Hector Sandoval \\
\hline National Institutes of Health & NS043124-11 & Hector Sandoval \\
\hline
\end{tabular}




\begin{tabular}{|c|c|c|}
\hline Funder & Grant reference number & Author \\
\hline $\begin{array}{l}\text { Research Education and Career } \\
\text { Horizon Institutional Research } \\
\text { and Academic Career Development } \\
\text { Award Fellowship }\end{array}$ & $\begin{array}{l}\text { 5K12GM084897 - Baylor } \\
\text { College of Medicine }\end{array}$ & Hector Sandoval \\
\hline National Institutes of Health & 5T32HD055200 & Vafa Bayat \\
\hline $\begin{array}{l}\text { Edward J. and Josephine G. Hudson } \\
\text { Scholarship }\end{array}$ & & Vafa Bayat \\
\hline Burroughs Wellcome Fund & 1008200 & Bo Xiong \\
\hline $\begin{array}{l}\text { National Science Council } \\
\text { Taiwan }\end{array}$ & $\begin{array}{l}\text { Taiwan Merit Scholarship } \\
\text { Program, NSC-095-SAF-I- } \\
\text { 564-015-TMS }\end{array}$ & Wu-Lin Charng \\
\hline Heiwa Nakajima Foundation & & Shinya Yamamoto \\
\hline Texas Children's Hospital & $\begin{array}{l}\text { Jan and Dan Duncan } \\
\text { Neurological Research } \\
\text { Institute }\end{array}$ & Shinya Yamamoto \\
\hline
\end{tabular}

The funders had no role in study design, data collection and interpretation, or the decision to submit the work for publication.

Author contributions

HS, Conception and design, Acquisition of data, Analysis and interpretation of data, Drafting or revising the article; C-KY, Made the Marf-transgenic and RNAi lines (Marf, Drp1 and dmiro) and established NMJ confocal microscopy assays; KC, Made the Marf-transgenic and established NMJ confocal microscopy assays; MJ, Designed and performed Drosophila X-chromosome screen (mutagenesis and mapping), Provided advice and insight during different stages of the project; TD, Performed the ETC enzymatic and aconitase assay; YQL, Performed the electrophysiology assays; VB, BX, KZ, GD, W-LC, SY, Designed and performed Drosophila X-chromosome screen (mutagenesis and mapping), Drafting or revising the article; LD, Performed sections and stains for TEM; BHG, Provided advice and insight during different stages of the project; HJB, Conception and design, Analysis and interpretation of data, Drafting or revising the article

\section{References}

Alexander C, Votruba M, Pesch UE, Thiselton DL, Mayer S, Moore A, Rodriguez M, Kellner U, Leo-Kottler B, Auburger G, Bhattacharya SS, Wissinger B. 2000. OPA1, encoding a dynamin-related GTPase, is mutated in autosomal dominant optic atrophy linked to chromosome 3q28. Nature Genetics 26:211-215. doi: 10.1038/79944.

Baqri RM, Turner BA, Rheuben MB, Hammond BD, Kaguni LS, Miller KE. 2009. Disruption of mitochondrial DNA replication in Drosophila increases mitochondrial fast axonal transport in vivo. PLOS ONE 4:e7874. doi: 10.1371/ journal.pone.0007874.

Bellen H, Budnik V. 2000. The Neuromuscular Junction. New York: Cold Spring Harbor Laboratory Press.

Chan DC. 2012. Fusion and fission: interlinked processes critical for mitochondrial health. Annual Review of Genetics 46:265-287. doi: 10.1146/annurev-genet-110410-132529.

Chavez VM, Marques G, Delbecque JP, Kobayashi K, Hollingsworth M, Burr J, Natzle JE, O'Connor MB. 2000. The Drosophila disembodied gene controls late embryonic morphogenesis and codes for a cytochrome P450 enzyme that regulates embryonic ecdysone levels. Development 127:4115-4126.

Chen H, Chomyn A, Chan DC. 2005. Disruption of fusion results in mitochondrial heterogeneity and dysfunction. The Journal of Biological Chemistry 280:26185-26192. doi: 10.1074/jbc.M503062200.

Chen H, Detmer SA, Ewald AJ, Griffin EE, Fraser SE, Chan DC. 2003. Mitofusins Mfn1 and Mfn2 coordinately regulate mitochondrial fusion and are essential for embryonic development. The Journal of Cell Biology 160:189-200. doi: 10.1083/jcb.200211046.

Chen H, McCaffery JM, Chan DC. 2007. Mitochondrial fusion protects against neurodegeneration in the cerebellum. Cell 130:548-562. doi: 10.1016/j.cell.2007.06.026.

Cipolat S, Martins de Brito O, Dal Zilio B, Scorrano L. 2004. OPA1 requires mitofusin 1 to promote mitochondrial fusion. Proceedings of the National Academy of Sciences of USA 101:15927-15932. doi: 10.1073/pnas. 0407043101.

Clark AJ, Block K. 1959. The absence of sterol synthesis in insects. The Journal of Biological Chemistry 234:2578-2582.

Cook RK, Christensen SJ, Deal JA, Coburn RA, Deal ME, Gresens JM, Kaufman TC, Cook KR. 2012. The generation of chromosomal deletions to provide extensive coverage and subdivision of the Drosophila melanogaster genome. Genome Biology 13:R21. doi: 10.1186/gb-2012-13-3-r21. 
Daniels RW, Collins CA, Gelfand MV, Dant J, Brooks ES, Krantz DE, Diantonio A. 2004. Increased expression of the Drosophila vesicular glutamate transporter leads to excess glutamate release and a compensatory decrease in quantal content. The Journal of Neuroscience 24:10466-10474. doi: 10.1523/JNEUROSCI.3001-04.2004.

Das N, Levine RL, Orr WC, Sohal RS. 2001. Selectivity of protein oxidative damage during aging in Drosophila melanogaster. The Biochemical Journal 360:209-216. doi: 10.1042/0264-6021:3600209.

de Brito OM, Scorrano L. 2008. Mitofusin 2 tethers endoplasmic reticulum to mitochondria. Nature 456:605-610. doi: 10.1038/nature07534.

Debattisti V, Pendin D, Ziviani E, Daga A, Scorrano L. 2014. Reduction of endoplasmic reticulum stress attenuates the defects caused by Drosophila mitofusin depletion. The Journal of Cell Biology 204:303-312. doi: $10.1083 /$ jcb. 201306121.

Debattisti V, Scorrano L. 2012. D. melanogaster, mitochondria and neurodegeneration: small model organism, big discoveries. Molecular and Cellular Neurosciences 55:77-86. doi: 10.1016/j.mcn.2012.08.007.

Delettre C, Lenaers G, Griffoin JM, Gigarel N, Lorenzo C, Belenguer P, Pelloquin L, Grosgeorge J, Turc-Carel C, Perret E, Astarie-Dequeker C, Lasquellec L, Arnaud B, Ducommun B, Kaplan J, Hamel CP. 2000. Nuclear gene OPA1, encoding a mitochondrial dynamin-related protein, is mutated in dominant optic atrophy. Nature Genetics 26:207-210. doi: 10.1038/79936.

Deng H, Dodson MW, Huang H, Guo M. 2008. The Parkinson's disease genes pink1 and parkin promote mitochondrial fission and/or inhibit fusion in Drosophila. Proceedings of the National Academy of Sciences of USA 105:14503-14508. doi: 10.1073/pnas.0803998105.

Di Cara F, King-Jones K. 2013. How clocks and hormones act in concert to control the timing of insect development. Current Topics in Developmental Biology 105:1-36. doi: 10.1016/B978-0-12-396968-2.00001-4.

Dickman DK, Horne JA, Meinertzhagen IA, Schwarz TL. 2005. A slowed classical pathway rather than kiss-andrun mediates endocytosis at synapses lacking synaptojanin and endophilin. Cell 123:521-533. doi: 10.1016/j. cell.2005.09.026.

Dickman DK, Lu Z, Meinertzhagen IA, Schwarz TL. 2006. Altered synaptic development and active zone spacing in endocytosis mutants. Current Biology 16:591-598. doi: 10.1016/j.cub.2006.02.058.

Dorn GW II, Clark CF, Eschenbacher WH, Kang MY, Engelhard JT, Warner SJ, Matkovich SJ, Jowdy CC. 2011. MARF and Opa1 control mitochondrial and cardiac function in Drosophila. Circulation Research 108:12-17. doi: 10.1161/CIRCRESAHA.110.236745.

Duarte A, Poderoso C, Cooke M, Soria G, Cornejo Maciel F, Gottifredi V, Podesta EJ. 2012. Mitochondrial fusion is essential for steroid biosynthesis. PLOS ONE 7:e45829. doi: 10.1371/journal.pone.0045829.

Emptage MH, Dreyers JL, Kennedy MC, Beinert H. 1983. Optical and EPR characterization of different species of active and inactive aconitase. The Journal of Biological Chemistry 258:11106-11111.

English AR, Voeltz GK. 2013. Endoplasmic reticulum structure and interconnections with other organelles. Cold Spring Harbor Perspectives in Biology 5:a013227. doi: 10.1101/cshperspect.a013227.

Frank S, Gaume B, Bergmann-Leitner ES, Leitner WW, Robert EG, Catez F, Smith CL, Youle RJ. 2001. The role of dynamin-related protein 1, a mediator of mitochondrial fission, in apoptosis. Developmental Cell 1:515-525. doi: 10.1016/S1534-5807(01)00055-7.

Frezza C, Cipolat S, Martins de Brito O, Micaroni M, Beznoussenko GV, Rudka T, Bartoli D, Polishuck RS, Danial NN, de Strooper B, Scorrano L. 2006. OPA1 controls apoptotic cristae remodeling independently from mitochondrial fusion. Cell 126:177-189. doi: 10.1016/j.cell.2006.06.025.

Gilbert LI. 2004. Halloween genes encode P450 enzymes that mediate steroid hormone biosynthesis in Drosophila melanogaster. Molecular and Cellular Endocrinology 215:1-10. doi: 10.1016/j.mce. 2003.11.003.

Gonzalez-Gaitan M, Jackle H. 1997. Role of Drosophila alpha-adaptin in presynaptic vesicle recycling. Cell 88:767-776. doi: 10.1016/S0092-8674(00)81923-6.

Graham BH, Li Z, Alesii EP, Versteken P, Lee C, Wang J, Craigen WJ. 2010. Neurologic dysfunction and male infertility in Drosophila porin mutants: a new model for mitochondrial dysfunction and disease. The Journal of Biological Chemistry 285:11143-11153. doi: 10.1074/jbc.M109.080317.

Greenspan P, Mayer EP, Fowler SD. 1985. Nile red: a selective fluorescent stain for intracellular lipid droplets. The Journal of Cell Biology 100:965-973. doi: 10.1083/jcb.100.3.965.

Guo X, Macleod GT, Wellington A, Hu F, Panchumarthi S, Schoenfield M, Marin L, Charlton MP, Atwood HL, Zinsmaier KE. 2005. The GTPase dMiro is required for axonal transport of mitochondria to Drosophila synapses. Neuron 47:379-393. doi: 10.1016/j.neuron.2005.06.027.

Haelterman NA, Yoon WH, Sandoval H, Jaiswal M, Shulman JM, Bellen HJ. 2014. A mitocentric view of Parkinson's disease. Annual Review of Neuroscience 37:137-159. doi: 10.1146/annurev-neuro-071013-014317.

Huang X, Warren JT, Gilbert LI. 2008. New players in the regulation of ecdysone biosynthesis. Journal of Genetics and Genomics 35:1-10. doi: 10.1016/S1673-8527(08)60001-6.

Issop L, Rone MB, Papadopoulos V. 2012. Organelle plasticity and interactions in cholesterol transport and steroid biosynthesis. Molecular and Cellular Endocrinology 371:34-46. doi: 10.1016/j.mce.2012.12.003.

Itoh K, Nakamura K, lijima M, Sesaki H. 2012. Mitochondrial dynamics in neurodegeneration. Trends in Cell Biology 23:64-71. doi: 10.1016/j.tcb.2012.10.006.

Koh TW, Korolchuk VI, Wairkar YP, Jiao W, Evergren E, Pan H, Zhou Y, Venken KJ, Shupliakov O, Robinson IM, O'Kane CJ, Bellen HJ. 2007. Eps15 and Dap160 control synaptic vesicle membrane retrieval and synapse development. The Journal of Cell Biology 178:309-322. doi: 10.1083/jcb.200701030.

Koh TW, Verstreken P, Bellen HJ. 2004. Dap160/intersectin acts as a stabilizing scaffold required for synaptic development and vesicle endocytosis. Neuron 43:193-205. doi: 10.1016/j.neuron.2004.06.029. 
Koopman WJ, Distelmaier F, Smeitink JA, Willems PH. 2013. OXPHOS mutations and neurodegeneration. The EMBO Journal 32:9-29. doi: 10.1038/emboj.2012.300.

Li Z, Okamoto K, Hayashi Y, Sheng M. 2004. The importance of dendritic mitochondria in the morphogenesis and plasticity of spines and synapses. Cell 119:873-887. doi: 10.1016/j.cell.2004.11.003.

Ly CV, Yao CK, Verstreken P, Ohyama T, Bellen HJ. 2008. straightjacket is required for the synaptic stabilization of cacophony, a voltage-gated calcium channel alpha1 subunit. The Journal of Cell Biology 181:157-170. doi: $10.1083 /$ jcb.200712152.

McBrayer Z, Ono H, Shimell M, Parvy JP, Beckstead RB, Warren JT, Thummel CS, Dauphin-Villemant C, Gilbert LI, O'Connor MB. 2007. Prothoracicotropic hormone regulates developmental timing and body size in Drosophila. Developmental Cell 13:857-871. doi: 10.1016/j.devcel.2007.11.003.

Miller DL, Ballard SL, Ganetzky B. 2012. Analysis of synaptic growth and function in Drosophila with an extended larval stage. The Journal of Neuroscience 32:13776-13786. doi: 10.1523/JNEUROSCI.0508-12.2012.

Miller WL. 2013. Steroid hormone synthesis in mitochondria. Molecular and Cellular Endocrinology 379:62-73. doi: 10.1016/j.mce.2013.04.014.

Mirth C, Truman JW, Riddiford LM. 2005. The role of the prothoracic gland in determining critical weight for metamorphosis in Drosophila melanogaster. Current Biology 15:1796-1807. doi: 10.1016/j.culb.2005.09.017.

Mishra P, Carelli V, Manfredi G, Chan DC. 2014. Proteolytic cleavage of opa1 stimulates mitochondrial inner membrane fusion and couples fusion to oxidative phosphorylation. Cell Metabolism 19:630-641. doi: 10.1016/j. cmet.2014.03.011.

Misko A, Jiang S, Wegorzewska I, Milbrandt J, Baloh RH. 2010. Mitofusin 2 is necessary for transport of axonal mitochondria and interacts with the Miro/Milton complex. The Journal of Neuroscience 30:4232-4240. doi: 10.1523/JNEUROSCI.6248-09.2010.

Munoz JP, Ivanova S, Sanchez-Wandelmer J, Martinez-Cristobal P, Noguera E, Sancho A, Diaz-Ramos A, Hernandez-Alvarez MI, Sebastian D, Mauvezin C, Palacin M, Zorzano A. 2013. Mfn2 modulates the UPR and mitochondrial function via repression of PERK. The EMBO Journal 32:2348-2361. doi: 10.1038/ emboj.2013.168.

Ngoh GA, Papanicolaou KN, Walsh K. 2012. Loss of mitofusin 2 promotes endoplasmic reticulum stress. The Journal of Biological Chemistry 287:20321-20332. doi: 10.1074/jbc.M112.359174.

Ohyama T, Verstreken P, Ly CV, Rosenmund T, Rajan A, Tien AC, Haueter C, Schulze KL, Bellen HJ. 2007. Huntingtin-interacting protein 14, a palmitoyl transferase required for exocytosis and targeting of CSP to synaptic vesicles. The Journal of Cell Biology 179:1481-1496. doi: 10.1083/jcb.200710061.

Park J, Lee SB, Lee S, Kim Y, Song S, Kim S, Bae E, Kim J, Shong M, Kim JM, Chung J. 2006. Mitochondrial dysfunction in Drosophila PINK1 mutants is complemented by parkin. Nature 441:1157-1161. doi: 10.1038/ nature04788.

Parks AL, Cook KR, Belvin M, Dompe NA, Fawcett R, Huppert K, Tan LR, Winter CG, Bogart KP, Deal JE, Deal-Herr ME, Grant D, Marcinko M, Miyazaki WY, Robertson S, Shaw KJ, Tabios M, Vysotskaia V, Zhao L, Andrade RS, Edgar KA, Howie E, Killpack K, Milash B, Norton A, Thao D, Whittaker K, Winner MA, Friedman L, Margolis J, Singer MA, Kopczynski C, Curtis D, Kaufman TC, Plowman GD, Duyk G, Francis-Lang HL. 2004. Systematic generation of high-resolution deletion coverage of the Drosophila melanogaster genome. Nature Genetics 36:288-292. doi: 10.1038/ng1312.

Parnas D, Haghighi AP, Fetter RD, Kim SW, Goodman CS. 2001. Regulation of postsynaptic structure and protein localization by the Rho-type guanine nucleotide exchange factor dPix. Neuron 32:415-424. doi: 10.1016/ S0896-6273(01)00485-8.

Pascual N, Belles X, Delbecque JP, Hua YJ, Koolman J. 1995. Quantification of ecdysteroids by immunoassay: comparison of enzyme immunoassay and radioimmunoassay. Zeitschrift für Naturforschung C 50:862-867.

Pilling AD, Horiuchi D, Lively CM, Saxton WM. 2006. Kinesin-1 and dynein are the primary motors for fast transport of mitochondria in Drosophila motor axons. Molecular Biology of the Cell 17:2057-2068. doi: 10.1091/ mbc.E05-06-0526.

Poole AC, Thomas RE, Yu S, Vincow ES, Pallanck L. 2010. The mitochondrial fusion-promoting factor mitofusin is a substrate of the PINK1/parkin pathway. PLOS ONE 5:e10054. doi: 10.1371/journal.pone.0010054.

Porcheron P, Foucrier J, Gros C, Pradelles P, Cassier P, Dray F. 1976. Radioimmunoassay of arthropod moulting hormone:beta-ecdysone antibodies production and 125 I-iodinated tracer preparation. FEBS Letters 61:159162. doi: 10.1016/0014-5793(76)81027-7.

Qin G, Schwarz T, Kittel RJ, Schmid A, Rasse TM, Kappei D, Ponimaskin E, Heckmann M, Sigrist SJ. 2005. Four different subunits are essential for expressing the synaptic glutamate receptor at neuromuscular junctions of Drosophila. The Journal of Neuroscience 25:3209-3218. doi: 10.1523/JNEUROSCI.4194-04.2005.

Rewitz KF, Yamanaka N, Gilbert LI, O'Connor MB. 2009. The insect neuropeptide PTTH activates receptor tyrosine kinase torso to initiate metamorphosis. Science 326:1403-1405. doi: 10.1126/science.1176450.

Rone MB, Midzak AS, Issop L, Rammouz G, Jagannathan S, Fan J, Ye X, Blonder J, Veenstra T, Papadopoulos V. 2012. Identification of a dynamic mitochondrial protein complex driving cholesterol import, trafficking, and metabolism to steroid hormones. Molecular Endocrinology 26:1868-1882. doi: 10.1210/ me.2012-1159.

Roos J, Kelly RB. 1998. Dap160, a neural-specific Eps15 homology and multiple SH3 domain-containing protein that interacts with Drosophila dynamin. The Journal of Biological Chemistry 273:19108-19119. doi: 10.1074/ jbc.273.30.19108.

Scaduto RC Jnr, Grotyohann LW. 1999. Measurement of mitochondrial membrane potential using fluorescent rhodamine derivatives. Biophysical Journal 76:469-477. doi: 10.1016/S0006-3495(99)77214-0. 
Scherer SS. 2011. CMT2A: the name doesn't tell the whole story. Neurology 76:1686-1687. doi: 10.1212/ WNL.0b013e31821bcc42.

Schuster CM, Ultsch A, Schloss P, Cox JA, Schmitt B, Betz H. 1991. Molecular cloning of an invertebrate glutamate receptor subunit expressed in Drosophila muscle. Science 254:112-114. doi: 10.1126/ science.1681587.

Sebastian D, Hernandez-Alvarez MI, Segales J, Sorianello E, Munoz JP, Sala D, Waget A, Liesa M, Paz JC, Gopalacharyulu P, Oresic M, Pich S, Burcelin R, Palacin M, Zorzano A. 2012. Mitofusin 2 (Mfn2) links mitochondrial and endoplasmic reticulum function with insulin signaling and is essential for normal glucose homeostasis. Proceedings of the National Academy of Sciences of USA 109:5523-5528. doi: 10.1073/pnas.1108220109.

Sheng ZH. 2014. Mitochondrial trafficking and anchoring in neurons: new insight and implications. The Journal of Cell Biology 204:1087-1098. doi: 10.1083/jcb.201312123.

Shidara Y, Hollenbeck PJ. 2010. Defects in mitochondrial axonal transport and membrane potential without increased reactive oxygen species production in a Drosophila model of Friedreich ataxia. The Journal of Neuroscience 30:11369-11378. doi: 10.1523/JNEUROSCI.0529-10.2010.

Stapleton M, Liao G, Brokstein P, Hong L, Carninci P, Shiraki T, Hayashizaki Y, Champe M, Pacleb J, Wan K, Yu C, Carlson J, George R, Celniker S, Rubin GM. 2002. The Drosophila gene collection: identification of putative full-length cDNAs for 70\% of D. melanogaster genes. Genome Research 12:1294-1300. doi: 10.1101/gr.269102.

Talamillo A, Sanchez J, Cantera R, Perez C, Martin D, Caminero E, Barrio R. 2008. Smt3 is required for Drosophila melanogaster metamorphosis. Development 135:1659-1668. doi: 10.1242/dev.020685.

van der Bliek AM, Shen Q, Kawajiri S. 2013. Mechanisms of mitochondrial fission and fusion. Cold Spring Harbor Perspectives in Biology 5:a011072. doi: 10.1101/cshperspect.a011072.

Venken KJ, Carlson JW, Schulze KL, Pan H, He Y, Spokony R, Wan KH, Koriabine M, de Jong PJ, White KP, Bellen HJ, Hoskins RA. 2009. Versatile P[acman] BAC libraries for transgenesis studies in Drosophila melanogaster. Nature Methods 6:431-434. doi: 10.1038/nmeth.1331.

Verstreken P, Kjaerulff O, Lloyd TE, Atkinson R, Zhou Y, Meinertzhagen IA, Bellen HJ. 2002. Endophilin mutations block clathrin-mediated endocytosis but not neurotransmitter release. Cell 109:101-112. doi: 10.1016/ S0092-8674(02)00688-8.

Verstreken P, Koh TW, Schulze KL, Zhai RG, Hiesinger PR, Zhou Y, Mehta SQ, Cao Y, Roos J, Bellen HJ. 2003. Synaptojanin is recruited by endophilin to promote synaptic vesicle uncoating. Neuron 40:733-748. doi: 10.1016/S0896-6273(03)00644-5.

Verstreken P, Ly CV, Venken KJ, Koh TW, Zhou Y, Bellen HJ. 2005. Synaptic mitochondria are critical for mobilization of reserve pool vesicles at Drosophila neuromuscular junctions. Neuron 47:365-378. doi: 10.1016/j. neuron.2005.06.018.

Verstreken P, Ohyama T, Bellen HJ. 2008. FM 1-43 labeling of synaptic vesicle pools at the Drosophila neuromuscular junction. Methods in Molecular Biology 440:349-369. doi: 10.1007/978-1-59745-178-9_26.

Wagh DA, Rasse TM, Asan E, Hofbauer A, Schwenkert I, Durrbeck H, Buchner S, Dabauvalle MC, Schmidt M, Qin G, Wichmann C, Kittel R, Sigrist SJ, Buchner E. 2006. Bruchpilot, a protein with homology to ELKS/CAST, is required for structural integrity and function of synaptic active zones in Drosophila. Neuron 49:833-844. doi: 10.1016/j.neuron.2006.02.008.

Walther TC, Farese RV Jnr. 2012. Lipid droplets and cellular lipid metabolism. Annual Review of Biochemistry 81:687-714. doi: 10.1146/annurev-biochem-061009-102430.

Wang T, Montell C. 2007. Phototransduction and retinal degeneration in Drosophila. Pflugers Archiv 454:821-847. doi: 10.1007/s00424-007-0251-1.

Warren JT, Petryk A, Marques G, Jarcho M, Parvy JP, Dauphin-Villemant C, O'Connor MB, Gilbert LI. 2002. Molecular and biochemical characterization of two P450 enzymes in the ecdysteroidogenic pathway of Drosophila melanogaster. Proceedings of the National Academy of Sciences of USA 99:11043-11048. doi: 10.1073/pnas.162375799.

Wasilewski M, Semenzato M, Rafelski SM, Robbins J, Bakardjiev Al, Scorrano L. 2012. Optic atrophy 1-dependent mitochondrial remodeling controls steroidogenesis in trophoblasts. Current Biology 22:1228-1234. doi: 10.1016/j. cub.2012.04.054.

Waterham HR, Koster J, van Roermund CW, Mooyer PA, Wanders RJ, Leonard JV. 2007. A lethal defect of mitochondrial and peroxisomal fission. The New England Journal of Medicine 356:1736-1741. doi: 10.1056/ NEJMoa064436.

Westermann B. 2009. Mitochondrial dynamics in model organisms: what yeasts, worms and flies have taught us about fusion and fission of mitochondria. Seminars in Cell \& Developmental Biology 21:542-549. doi: 10.1016/j. semcdb.2009.12.003.

Xiong B, Bayat V, Jaiswal M, Zhang K, Sandoval H, Charng WL, Li T, David G, Duraine L, Lin YQ, Neely GG, Yamamoto S, Bellen HJ. 2012. Crag is a GEF for Rab11 required for rhodopsin trafficking and maintenance of adult photoreceptor cells. PLOS Biology 10:e1001438. doi: 10.1371/journal.pbio.1001438.

Yamamoto S, Jaiswal M, Charng WL, Gambin T, Karaca E, Mirzaa G, Wiszniewski W, Sandoval H, Haelterman NA, Xiong B, Zhang K, Bayat V, David G, Li T, Chen K, Gala U, Harel T, Pehlivan D, Penney S, Vissers LE, de Ligt J, Jhangiani SN, Xie Y, Tsang SH, Parman Y, Sivaci M, Battaloglu E, Muzny D, Wan YW, Liu Z, Lin-Moore AT, Clark RD, Curry CJ, Link N, Schulze KL, Boerwinkle E, Dobyns WB, Allikmets R, Gibbs RA, Chen R, Lupski JR, Wangler MF, Bellen HJ. 2014. A Drosophila genetic resource of mutants to study mechanisms underlying human genetic diseases. Cell 159:200-214. doi: 10.1016/j.cell.2014.09.002.

Yamanaka N, Rewitz KF, O'Connor MB. 2012. Ecdysone control of developmental transitions: lessons from Drosophila research. Annual Review of Entomology 58:497-516. doi: 10.1146/annurev-ento-120811-153608. 
Yan LJ, Levine RL, Sohal RS. 1997. Oxidative damage during aging targets mitochondrial aconitase. Proceedings of the National Academy of Sciences of USA 94:11168-11172. doi: 10.1073/pnas.94.21.11168.

Yao CK, Lin YQ, Ly CV, Ohyama T, Haueter CM, Moiseenkova-Bell VY, Wensel TG, Bellen HJ. 2009. A synaptic vesicle-associated $\mathrm{Ca} 2+$ channel promotes endocytosis and couples exocytosis to endocytosis. Cell 138:947-960. doi: 10.1016/j.cell.2009.06.033.

Yao JG, Weasner BM, Wang LH, Jang CC, Weasner B, Tang CY, Salzer CL, Chen CH, Hay B, Sun YH, Kumar JP. 2008. Differential requirements for the Pax6(5a) genes eyegone and twin of eyegone during eye development in Drosophila. Developmental Biology 315:535-551. doi: 10.1016/j.ydbio.2007.12.037.

Yu-Wai-Man P, Griffiths PG, Gorman GS, Lourenco CM, Wright AF, Auer-Grumbach M, Toscano A, Musumeci O, Valentino ML, Caporali L, Lamperti C, Tallaksen CM, Duffey P, Miller J, Whittaker RG, Baker MR, Jackson MJ, Clarke MP, Dhillon B, Czermin B, Stewart JD, Hudson G, Reynier P, Bonneau D, Marques W Jnr, Lenaers G, Mcfarland R, Taylor RW, Turnbull DM, Votruba M, Zeviani M, Carelli V, Bindoff LA, Horvath R, Amati-Bonneau P, Chinnery PF. 2010. Multi-system neurological disease is common in patients with OPA1 mutations. Brain 133:771-786. doi: 10.1093/brain/awq007.

Zhang K, Li Z, Jaiswal M, Bayat V, Xiong B, Sandoval H, Charng WL, David G, Haueter C, Yamamoto S, Graham BH, Bellen HJ. 2013. The C8ORF38 homologue Sicily is a cytosolic chaperone for a mitochondrial complex I subunit. The Journal of Cell Biology 200:807-820. doi: 10.1083/jcb.201208033.

Ziviani E, Tao RN, Whitworth AJ. 2010. Drosophila parkin requires PINK1 for mitochondrial translocation and ubiquitinates mitofusin. Proceedings of the National Academy of Sciences of USA 107:5018-5023. doi: 10.1073/ pnas.0913485107.

Zuchner S, Mersiyanova IV, Muglia M, Bissar-Tadmouri N, Rochelle J, Dadali EL, Zappia M, Nelis E, Patitucci A, Senderek J, Parman Y, Evgrafov O, Jonghe PD, Takahashi Y, Tsuji S, Pericak-Vance MA, Quattrone A, Battaloglu E, Polyakov AV, Timmerman V, Schroder JM, Vance JM. 2004. Mutations in the mitochondrial GTPase mitofusin 2 cause Charcot-Marie-Tooth neuropathy type 2A. Nature Genetics 36:449-451. doi: 10.1038/ng1341. 